\title{
A crack perpendicular to the bimaterial interface in finite solid
}

\author{
S.H. Chen ${ }^{\mathrm{a}, *}$, T.C. Wang ${ }^{\mathrm{a}}$, Sharon Kao-Walter ${ }^{\mathrm{b}}$ \\ ${ }^{\text {a }}$ LNM, Institute of Mechanics, Chinese Academy of Sciences, Beijing 100080, China \\ ${ }^{\mathrm{b}}$ Department of Mechanical Engineering, Blekinge Institute of Technology, S-371 79 Karlskrona, Sweden
}

Received 19 July 2002; received in revised form 3 January 2003

\begin{abstract}
The dislocation simulation method is used in this paper to derive the basic equations for a crack perpendicular to the bimaterial interface in a finite solid. The complete solutions to the problem, including the $T$ stress and the stress intensity factors are obtained. The stress field characteristics are investigated in detail. It is found that when the crack is within a weaker material, the stress intensity factor is smaller than that in a homogeneous material and it decreases when the distance between the crack tip and interface decreases. When the crack is within a stiffer material, the stress intensity factor is larger than that in a homogeneous material and it increases when the distance between the crack tip and interface decreases. In both cases, the stress intensity factor will increase when the ratio of the size of a sample to the crack length decreases. A comparison of stress intensity factors between a finite problem and an infinite problem has been given also. The stress distribution ahead of the crack tip, which is near the interface, is shown in details and the $T$ stress effect is considered.
\end{abstract}

(c) 2003 Elsevier Science Ltd. All rights reserved.

Keywords: Finite bimaterial solid; Interface; Crack; Stress intensity factor; $T$ stress

\section{Introduction}

The influence of cracks is very important in advanced materials, such as fiber or particle reinforced composites, metal and ceramics interfaces, laminated ceramics, packaging materials and so on. Interface failures are common features in those materials and thin films. The design process of those components requires a better understanding of the failure mechanisms. An important task is to study in detail the fracture characteristics of cracks along or perpendicular to the interface.

Many researchers have investigated the interaction between an interface and a crack with various methods. Zak and Williams (1963) used the eigenfunction expansion method to analyze the stress singularity at the tip of a crack, perpendicular to and terminating at the interface. Cook and Erdogan (1972) used the Mellin transform method to derive the governing equation of finite cracks perpendicular to an interface and obtained the stress intensity factors. Erdogan and Biricikoglu (1973) solved the problem of two

\footnotetext{
${ }^{*}$ Corresponding author. Tel.: +86-10-6254-5533x3023; fax: +86-10-6256-1284.

E-mail address: chenshaohua72@hotmail.com (S.H. Chen).
} 
bounded half planes with a crack going through the interface. Bogy (1971) investigated the stress singularity of an infinite crack terminated at the interface with an arbitrary angle. Wang and Chen (1993) used photoelasticity to determine the stress distribution and the stress intensity of a crack perpendicular to the interface. Wang and Ståhle (1998a,b) used the dislocation simulation approach to investigate a crack perpendicular to and terminating at the bimaterial interface. Lin and Mar (1976) presented a finite element analysis of the stress intensity factors for cracks perpendicular to the bimaterial interface. Meguid et al. (1995) proposed a novel finite element to analyze edge cracks in a finite elastic homogeneous body and a finite crack perpendicular to the interface in an infinite bimaterial solid. Chen (1994) used the body force to determine the stress intensity factors for a crack normal to and terminated at the bimaterial interface. Suo (1989) analyzed the interaction problem of an edge dislocation with a bimaterial interface. Stahle et al. (1995) investigated a crack growing towards to a bimaterial interface, where they carried out an experiment work and a finite element simulation. Recently, Leblond and Frelat (2000) studied a crack kinking from an initially closed crack and Chen (2000) investigated the $T$ stress in plane elasticity crack problems.

All the above studies are almost all about crack and interface problems in an infinite body. Few analytical solutions about interaction of a crack and an interface in a finite solid are available. In engineering applications, one has to deal with small bodies, especially the interaction of a crack and an interface in a bimaterial solid or a packaging.

The dislocation simulation approach is used in this paper to derive the basic equations for a crack perpendicular to the interface in a finite solid. The dislocation density is expressed as a series of the first Chebyshev polynominal with a set of unknown coefficients. Two additional holomorphic functions are introduced in order to satisfy the outside boundary conditions. Combined with the boundary collocation method, the governing equations are solved numerically and the complete solution of the problem and the stress intensity factors are obtained. Two kinds of cases with different loading forms are considered. The results are interesting and given in detail.

\section{Basic equations}

\subsection{Complex potentials}

In this section, basic equations are given for a finite crack perpendicular to the interface in an elastic finite body. Stresses and displacements can be expressed by two Muskhelishivili's potentials:

$$
\left\{\begin{array}{l}
\sigma_{x}+\sigma_{y}=4 \operatorname{Re}\{\Phi(z)\} \\
\sigma_{y}-\mathrm{i} \tau_{x y}=\Phi(z)+\Omega(\bar{z})+(z-\bar{z}) \overline{\Phi^{\prime}(z)} \\
2 \mu\left(u_{x}+\mathrm{i} u_{y}\right)=\kappa \phi(z)-\omega(\bar{z})-(z-\bar{z}) \overline{\Phi(z)}
\end{array}\right.
$$

where $\Phi(z)=\phi^{\prime}(z), \Omega(z)=\omega^{\prime}(z)$.

The complex potentials for an edge dislocation at $z=s$ in an infinite elastic solid can be expressed as follows:

$$
\begin{aligned}
& \Phi_{0}(z)=\frac{B}{z-s} \\
& \Omega_{0}(z)=\frac{B}{z-\bar{s}}+\bar{B} \frac{s-\bar{s}}{(z-\bar{s})^{2}}
\end{aligned}
$$

where $B=(\mu /(\pi \mathrm{i}(\kappa+1)))\left(b_{x}+\mathrm{i} b_{y}\right)$ and $b_{x}$ and $b_{y}$ are the $x$ and $y$ components of the Burgers vector of the dislocation, $\kappa=3-4 v$ for the plane strain case and $\kappa=(3-v) /(1+v)$ for the plane stress case, $v$ and $\mu$ are Poisson's ratio and shear modulus, respectively. 


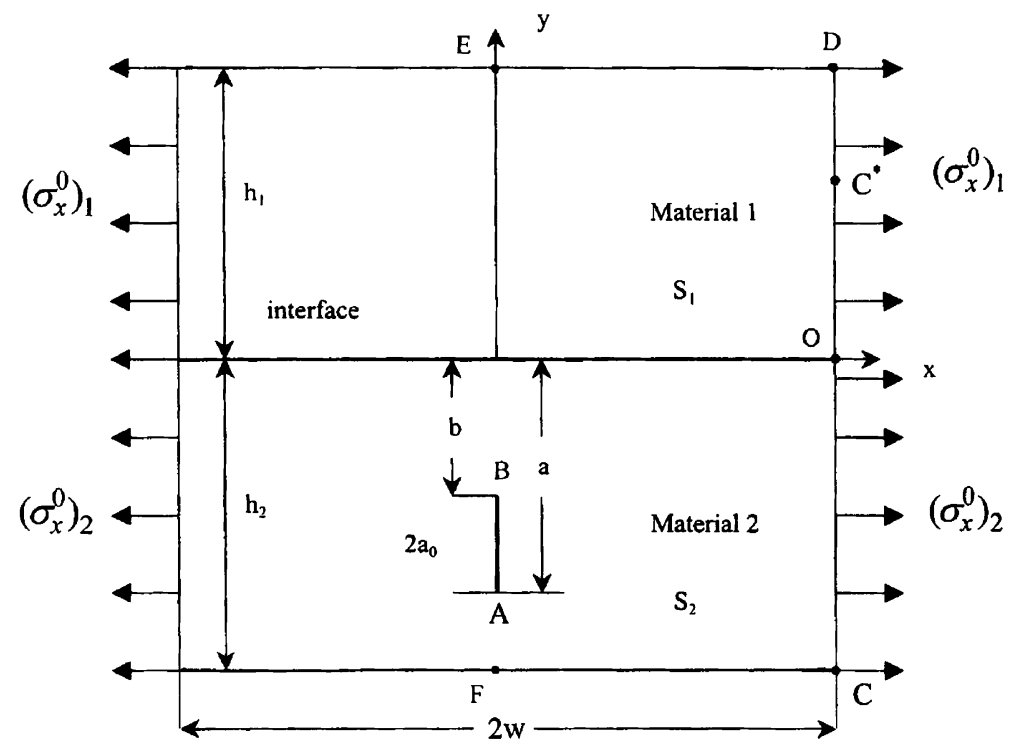

Fig. 1. A finite crack perpendicular to the bimaterial interface of a finite solid for case I.

The interaction of an edge dislocation with a bimaterial interface in an infinite solid was studied by Dundurs (1969), Suo (1989), Wang and Ståhle (1998a,b) among others. If the edge dislocation is embedded in material 2 as shown in Fig. 1, the complex potentials are (see Suo, 1989)

$$
\begin{gathered}
\Phi(z)= \begin{cases}\left(1+\Lambda_{1}\right) \Phi_{0}(z) & z \in S_{1} \\
\Phi_{0}(z)+\Lambda_{2} \Omega_{0}(z) & z \in S_{2}\end{cases} \\
\Omega(z)= \begin{cases}\Omega_{0}(z)+\Lambda_{1} \Phi_{0}(z) & z \in S_{1} \\
\left(1+\Lambda_{2}\right) \Omega_{0}(z) & z \in S_{2}\end{cases}
\end{gathered}
$$

where

$$
\Lambda_{1}=\frac{\alpha+\beta}{1-\beta} \quad \Lambda_{2}=\frac{\alpha-\beta}{1+\beta}
$$

and $\alpha$ and $\beta$ are two Dundur's parameters

$$
\alpha=\frac{\Gamma\left(\kappa_{2}+1\right)-\left(\kappa_{1}+1\right)}{\Gamma\left(\kappa_{2}+1\right)+\left(\kappa_{1}+1\right)} \quad \beta=\frac{\Gamma\left(\kappa_{2}-1\right)-\left(\kappa_{1}-1\right)}{\Gamma\left(\kappa_{2}+1\right)+\left(\kappa_{1}+1\right)}
$$

where

$$
\Gamma=\mu_{1} / \mu_{2}
$$

Considering the traction and displacement continuous conditions at the interface, one can get the complex potentials for the same problem but in a finite solid,

$$
\begin{aligned}
& \Phi(z)= \begin{cases}\left(1+\Lambda_{1}\right) \Phi_{0}(z)+\left(1+\Lambda_{1}\right) F(z)+\lambda_{1} G(z) & z \in S_{1} \\
\Phi_{0}(z)+\Lambda_{2} \Omega_{0}(z)+\lambda_{2} G(z)+F(z) & z \in S_{2}\end{cases} \\
& \Omega(z)= \begin{cases}\Omega_{0}(z)+\Lambda_{1} \Phi_{0}(z)+\Lambda_{1} F(z)+\lambda_{1} G(z) & z \in S_{1} \\
\left(1+\Lambda_{2}\right) \Omega_{0}(z)+\lambda_{2} G(z) & z \in S_{2}\end{cases}
\end{aligned}
$$


where

$$
\lambda_{1}=\frac{1}{1-\beta} \quad \lambda_{2}=\frac{1}{1+\beta}
$$

and two holomorphic functions $F, G$ in the finite solid can be expressed as follows:

$$
F(z)=\sum_{n=1}^{\infty} n b_{n} z^{n-1} \quad G(z)=\sum_{n=1}^{\infty} n c_{n} z^{n-1}
$$

The crack is considered as a continuous distribution of dislocations. So we have

$$
\begin{aligned}
& \Phi_{0}(z)=\frac{\mu_{2}}{\pi \mathrm{i}\left(1+\kappa_{2}\right)} \int_{b}^{a} \frac{\left(b_{x}+\mathrm{i} b_{y}\right)}{z+\mathrm{i} t} \mathrm{~d} t \\
& \Omega_{0}(z)=\frac{\mu_{2}}{\pi \mathrm{i}\left(1+\kappa_{2}\right)} \int_{b}^{a} \frac{\left(b_{x}+\mathrm{i} b_{y}\right)}{z-\mathrm{i} t} \mathrm{~d} t+\frac{2 \mu_{2}}{\pi\left(1+\kappa_{2}\right)} \int_{b}^{a} \frac{t\left(b_{x}-\mathrm{i} b_{y}\right)}{(z-\mathrm{i} t)^{2}} \mathrm{~d} t
\end{aligned}
$$

where $a$ and $b$ are shown in Fig. 1 and $c$ is the distance from the center of the crack to the interface, $a_{0}$ is a half of the crack length.

$$
a_{0}=(a-b) / 2 \quad c=(a+b) / 2
$$

According to Wang and Ståhle (1998a,b), we introduce a new complex variable $z^{*}$ and a new function $I\left(z^{*}\right)$

$$
z^{*}=\mathrm{i} z \quad I\left(z^{*}\right)=\frac{1}{\pi} \int_{b}^{a} \frac{b_{x}+\mathrm{i} b_{y}}{z^{*}-t} \mathrm{~d} t
$$

Using the following variable transformations:

$$
z^{*}=\frac{a+b}{2}+\frac{a-b}{2} \varsigma \quad t=\frac{a+b}{2}+\frac{a-b}{2} \xi
$$

the function $I\left(z^{*}\right)$ can be represented as

$$
I\left(z^{*}\right)=\frac{1}{\pi} \int_{-1}^{1} \frac{b_{x}+\mathrm{i} b_{y}}{\varsigma-\xi} \mathrm{d} \xi
$$

Assume that the dislocation density can be expanded as a series of the first Chebyshev polynomial

$$
b_{x}+\mathrm{i} b_{y}=\frac{1}{\sqrt{1-\xi^{2}}} \sum_{m=1}^{\infty} \alpha_{m} T_{m}(\xi)
$$

where $T_{m}(\xi)$ is the first Chebyshev polynomial

$$
T_{m}(\xi)=\cos m \theta \quad \xi=\cos \theta=(t-c) / a_{0}
$$

The opening displacement on the crack surface can be obtained

$$
\delta_{x}+\mathrm{i} \delta_{y}=\int_{b}^{t}\left(b_{x}+\mathrm{i} b_{y}\right) \mathrm{d} t=\int_{-1}^{\xi}\left[\frac{1}{\sqrt{1-\xi^{2}}} \sum_{m=0}^{\infty} \alpha_{m} T(\xi)\right] \mathrm{d} \xi a_{0}=a_{0} \alpha_{0}(\pi-\theta)-a_{0} \sum_{m=1}^{\infty} \alpha_{m} \frac{\sin m \theta}{m}
$$

At the crack tip A as shown in Fig. 1, we have $t=a, \theta=0$ and substituting them into the above equation, the following equation can be obtained:

$$
\alpha_{0}=0
$$


Using the following equation, which can be found in Gladwell (1980):

$$
\frac{1}{\pi} \int_{-1}^{1} \frac{T_{m}(x)}{\sqrt{1-x^{2}}(z-x)} \mathrm{d} x=\frac{1}{\sqrt{z^{2}-1}}\left[z-\sqrt{z^{2}-1}\right]^{m}
$$

We obtain

$$
I\left(z^{*}\right)=\frac{1}{\sqrt{\varsigma^{2}-1}} \sum_{m=0,1}^{\infty} \alpha_{m}\left[\varsigma-\sqrt{\varsigma^{2}-1}\right]^{m} \quad \varsigma=\frac{z^{*}-c}{a_{0}}
$$

\subsection{Stress jump across interface}

We know that the displacements should be continuous across the interface and the strain $\varepsilon_{x}$ should be continuous across the interface also. It follows that:

$$
\left(\varepsilon_{x}\right)_{1}=\left(\varepsilon_{x}\right)_{2} \text { on the interface }
$$

For the plane strain problem, the above equation can be written as

$$
\left(\sigma_{x}\right)_{1}=\frac{\mu_{1}\left(1-v_{2}\right)}{\mu_{2}\left(1-v_{1}\right)}\left(\sigma_{x}\right)_{2}+\frac{\sigma_{y}}{1-v_{1}}\left(v_{1}-\frac{\mu_{1}}{\mu_{2}} v_{2}\right) \text { on the interface }
$$

With the two Dundur's parameters, the above equation can be written as

$$
\left(\sigma_{x}\right)_{1}=\frac{(1+\alpha)}{(1-\alpha)}\left(\sigma_{x}\right)_{2}+\frac{2 \sigma_{y}}{1-\alpha}(2 \beta-\alpha) \quad \text { on the interface }
$$

We assume that the external loading satisfies Eq. (27), so

$$
\left(\sigma_{x}^{0}\right)_{1}=\frac{(1+\alpha)}{(1-\alpha)}\left(\sigma_{x}^{0}\right)_{2}+\frac{2 \sigma_{y}^{0}}{1-\alpha}(2 \beta-\alpha)
$$

where $\left(\sigma_{x}^{0}\right)_{1}$ and $\left(\sigma_{x}^{0}\right)_{2}$ are the external loading acted on the left and right boundaries in materials 1 and 2 in the direction of $x$-axis, respectively. $\sigma_{y}^{0}$ is the external loading acted on the upper and lower boundaries in the bimaterial in the direction of $y$-axis.

In the present paper, two kinds of loading forms are considered and we call them cases I and II.

\subsection{Governing equations for Case I}

Case I is a symmetric problem as shown in Fig. 1, in which $\left(\sigma_{x}^{0}\right)_{1}$ is loaded on the right and left boundaries of the upper material 1 and $\left(\sigma_{x}^{0}\right)_{2}$ is loaded on the right and left boundaries of the lower material 2. In this case $\sigma_{y}^{0}=0$ and the relation between $\left(\sigma_{x}^{0}\right)_{1}$ and $\left(\sigma_{x}^{0}\right)_{2}$ becomes

$$
\left(\sigma_{x}^{0}\right)_{1}=\frac{(1+\alpha)}{(1-\alpha)}\left(\sigma_{x}^{0}\right)_{2}
$$

The crack lies in the lower material 2 and the crack length is $2 a_{0}$. The distance between the crack tip B and the interface is $b$. The distance between another crack tip A and the interface is $a$. The width of the sample is $2 w$ and the height of the upper region $S_{1}$ is $h_{1}$ and that of the lower region $S_{2}$ is $h_{2}$. Both the upper and lower materials are elastic.

Since the problem is an elastic one, we use a superposition scheme and need two solutions. The first solution is that for the bimaterial subject to a uniform external loading on the outside boundaries and is a homogeneous solution,

$$
\sigma_{x}=\left(\sigma_{x}^{0}\right)_{1} \quad \sigma_{y}=\sigma_{y}^{0}=0 \quad \tau_{x y}=0 \quad \text { in material } 1
$$




$$
\sigma_{x}=\left(\sigma_{x}^{0}\right)_{2} \quad \sigma_{y}=\sigma_{y}^{0}=0 \quad \tau_{x y}=0 \quad \text { in material } 2
$$

The second solution is that for a crack perpendicular to the interface with the uniform traction prescribed on the crack faces and traction-free on the outside boundaries. So we have the following equation for the crack face:

$$
\sigma_{x}+\mathrm{i} \tau_{x y}=\Phi(z)+2 \overline{\Phi(z)}-\Omega(\bar{z})-(z-\bar{z}) \overline{\Phi^{\prime}(z)}=-\sigma \quad z= \pm 0+\mathrm{i} y \quad-a<y<-b
$$

where

$$
\sigma=\left(\sigma_{x}^{0}\right)_{2}
$$

Since it is a symmetric problem, that is, $b_{y}=0$, we obtain

$$
\begin{aligned}
& \Phi_{0}(z)=\frac{\mu_{2}}{\left(\kappa_{2}+1\right)} I(\mathrm{i} z) \\
& \Omega_{0}(z)=\frac{\mu_{2}}{\left(\kappa_{2}+1\right)} I(-\mathrm{i} z)-\frac{2 \mu_{2}}{\left(\kappa_{2}+1\right)} \mathrm{i} z I^{\prime}(-\mathrm{i} z)
\end{aligned}
$$

Substituting the above Eqs. (34), (35), (9), (10) and (12) into Eq. (32), we obtain the following traction equation on the crack face:

$$
\begin{aligned}
& \frac{\mu_{2}}{\kappa_{2}+1}\left\{2 \overline{I^{+}(t)}+2 t\left[\overline{I^{+}(t)}-I^{\prime-}(t)\right]+I^{+}(t)-I^{-}(t)+\left(3 \Lambda_{2}-\Lambda_{1}\right) I(-t)-12 \Lambda_{2} t I^{\prime}(-t)\right. \\
& \left.+4 t^{2} \Lambda_{2} I^{\prime \prime}(-t)\right\}+\sum_{n=1}^{\infty} n\left(b_{n}+\lambda_{2} c_{n}\right) z^{n-1}+2 \sum_{n=1}^{\infty} n\left(\bar{b}_{n}+\lambda_{2} \bar{c}_{n}\right) \bar{z}^{n-1}-\lambda_{1} \sum_{n=1}^{\infty} n c_{n} \bar{z}^{n-1} \\
& \quad-\Lambda_{1} \sum_{n=1}^{\infty} n b_{n} \bar{z}^{n-1}+2 \sum_{n=1}^{\infty} n(n-1)\left(\bar{b}_{n}+\lambda_{2} \bar{c}_{n}\right) \bar{z}^{n-1}=-\sigma
\end{aligned}
$$

where $b<t<a$.

Since it is a problem of finite solid, the boundary effect must be considered in the solution. In the present paper, we use the resultant forces on each boundary as boundary conditions. Point $\mathrm{O}$ is assumed to be fixed at all times, a point $\mathrm{C}^{*}$ is permitted to move. The boundary conditions in the present analysis can be written in terms of the resultant forces from $\mathrm{O}$ to $\mathrm{C}^{*}$ as follows:

$$
\begin{array}{ll}
\mathrm{C}^{*} \in \mathrm{OCF}: & X+\mathrm{i} Y=0 \\
\mathrm{C}^{*} \in \mathrm{ODE}: & X+\mathrm{i} Y=0
\end{array}
$$

The resultant forces from $\mathrm{O}$ to $\mathrm{C}^{*}$ can be expressed as

$$
X(z)+\mathrm{i} Y(z)=-\mathrm{i}[\phi(z)+\omega(\bar{z})+(z-\bar{z}) \overline{\Phi(z)}]_{\mathrm{O}}^{\mathrm{C}^{*}}
$$

where

$$
\begin{aligned}
\phi(z)= & \int \Phi(z) \mathrm{d} z \\
= & \left\{\begin{array}{l}
\frac{\left(1+\Lambda_{1}\right) \mu_{2}}{1+k_{2}} \sum_{m=1}^{\infty} \frac{\alpha_{m} a_{0} i}{m}\left[\varsigma-\sqrt{\varsigma^{2}-1}\right]^{m}+\left(1+\Lambda_{1}\right) \sum_{n=1}^{\infty} b_{n} z^{n}+\lambda_{1} \sum_{n=1}^{\infty} c_{n} z^{n}+b_{01}, \quad z \in S_{1} \\
\frac{\mu_{2}}{1+k_{2}} \sum_{m=1}^{\infty} \frac{\alpha_{m} a_{0} i}{m}\left[\varsigma-\sqrt{\varsigma^{2}-1}\right]^{m}+\frac{\Lambda_{2} \mu_{2}}{1+k_{2}} \sum_{m=1}^{\infty} \frac{\alpha_{m} a_{0} i}{m}\left[\varsigma_{1}-\sqrt{\varsigma_{1}^{2}-1}\right]^{m} \\
\quad+\frac{2 \Lambda_{2} \mu_{2}}{1+k_{2}} z I(-\mathrm{i} z)+\lambda_{2} \sum_{n=1}^{\infty} c_{n} z^{n}+\sum_{n=1}^{\infty} b_{n} z^{n}+b_{02}, \quad z \in S_{2}
\end{array}\right.
\end{aligned}
$$




$$
\omega(z)=\int \Omega(z) \mathrm{d} z=\left\{\begin{array}{l}
\frac{\Lambda_{1} \mu_{2}}{1+k_{2}} \sum_{m=1}^{\infty} \frac{\alpha_{m} a_{0} i}{m}\left[\varsigma-\sqrt{\varsigma^{2}-1}\right]^{m}+\frac{\mu_{2}}{1+k_{2}} \sum_{m=1}^{\infty} \frac{\alpha_{m} a_{0} i}{m}\left[\varsigma_{1}-\sqrt{\varsigma_{1}^{2}-1}\right]^{m} \\
\quad+\frac{2 \mu_{2}}{1+k_{2}} z I(-\mathrm{i} z)+\Lambda_{1} \sum_{n=1}^{\infty} b_{n} z^{n}+\lambda_{1} \sum_{n=1}^{\infty} c_{n} z^{n}+c_{01}, \quad z \in S_{1} \\
\frac{\left(1+\Lambda_{2}\right) \mu_{2}}{1+k_{2}} \sum_{m=1}^{\infty} \frac{\alpha_{m} a_{0} i}{m}\left[\varsigma_{1}-\sqrt{\varsigma_{1}^{2}-1}\right]^{m} \\
+\frac{2\left(1+\Lambda_{2}\right) \mu_{2}}{1+k_{2}} z I(-\mathrm{i} z)+\lambda_{2} \sum_{n=1}^{\infty} c_{n} z^{n}+c_{02}, \quad z \in S_{2}
\end{array}\right.
$$

where $b_{01}, b_{02}, c_{01}, c_{02}$ are the unknown coefficients to be determined, which are related with the rigid displacements and

$$
\varsigma=\frac{\mathrm{i} z-c}{a_{0}} \quad \varsigma_{1}=\frac{-\mathrm{i} z-c}{a_{0}}
$$

On the boundaries $\mathrm{OC}$ and $\mathrm{CF}$ as shown in Fig. 1, we have

$$
\begin{aligned}
\phi(z)= & \frac{\mu_{2}}{1+k_{2}} \sum_{m=1}^{\infty} \frac{\alpha_{m} a_{0} i}{m}\left[\varsigma-\sqrt{\varsigma^{2}-1}\right]^{m}+\frac{\Lambda_{2} \mu_{2}}{1+k_{2}} \sum_{m=1}^{\infty} \frac{\alpha_{m} a_{0} i}{m}\left[\varsigma_{1}-\sqrt{\varsigma_{1}^{2}-1}\right]^{m} \\
& +\frac{2 \Lambda_{2} \mu_{2}}{1+k_{2}} z I(-\mathrm{i} z)+\lambda_{2} \sum_{n=1}^{\infty} c_{n} z^{n}+\sum_{n=1}^{\infty} b_{n} z^{n}+b_{02} \\
\omega(\bar{z})= & \frac{\Lambda_{1} \mu_{2}}{1+k_{2}} \sum_{m=1}^{\infty} \frac{\alpha_{m} a_{0} i}{m}\left[\varsigma_{2}-\sqrt{\varsigma_{2}^{2}-1}\right]^{m}+\frac{\mu_{2}}{1+k_{2}} \sum_{m=1}^{\infty} \frac{\alpha_{m} a_{0} i}{m}\left[\varsigma_{3}-\sqrt{\varsigma_{3}^{2}-1}\right]^{m} \\
& +\frac{2 \mu_{2}}{1+k_{2}} \bar{z} I(-\mathrm{i} \bar{z})+\Lambda_{1} \sum_{n=1}^{\infty} b_{n} \bar{z}^{n}+\lambda_{1} \sum_{n=1}^{\infty} c_{n} \bar{z}^{n}+c_{01} \\
\overline{\Phi(z)}= & \frac{\mu_{2}}{1+k_{2}} \overline{I(\mathrm{i} z)}+\frac{\Lambda_{2} \mu_{2}}{1+k_{2}} \overline{I(-\mathrm{i} z)}+\frac{2 \Lambda_{2} \mu_{2}}{1+k_{2}} \overline{\mathrm{z}} \overline{I^{\prime}(-\mathrm{i} z)}+\lambda_{2} \sum_{n=1}^{\infty} n \bar{c}_{n} \bar{z}^{n-1}+\sum_{n=1}^{\infty} n \bar{b}_{n} \bar{z}^{n-1}
\end{aligned}
$$

where

$$
\varsigma_{2}=\frac{\mathrm{i} \bar{z}-c}{a_{0}} \quad \varsigma_{3}=\frac{-\mathrm{i} \bar{z}-c}{a_{0}}
$$

On the boundaries OD and DE as shown in Fig. 1,

$$
\begin{aligned}
& \phi(z)=\frac{\left(1+\Lambda_{1}\right) \mu_{2}}{1+k_{2}} \sum_{m=1}^{\infty} \frac{\alpha_{m} a_{0} i}{m}\left[\varsigma-\sqrt{\varsigma^{2}-1}\right]^{m}+\left(1+\Lambda_{1}\right) \sum_{n=1}^{\infty} b_{n} z^{n}+\lambda_{1} \sum_{n=1}^{\infty} c_{n} z^{n}+b_{01} \\
& \omega(\bar{z})=\frac{\left(1+\Lambda_{2}\right) \mu_{2}}{1+k_{2}} \sum_{m=1}^{\infty} \frac{\alpha_{m} a_{0} i}{m}\left[\varsigma_{3}-\sqrt{\varsigma_{3}^{2}-1}\right]^{m}+\frac{2\left(1+\Lambda_{2}\right) \mu_{2}}{1+k_{2}} \bar{z} I(-\mathrm{i} \bar{z})+\lambda_{2} \sum_{n=1}^{\infty} c_{n} \bar{z}^{n}+c_{02} \\
& \overline{\Phi(z)}=\frac{\left(1+\Lambda_{1}\right) \mu_{2}}{1+k_{2}} \overline{I(\mathrm{i} z)}+\left(1+\Lambda_{1}\right) \sum_{n=1}^{\infty} n \bar{b}_{n} \bar{z}^{n-1}+\lambda_{1} \sum_{n=1}^{\infty} n \bar{c}_{n} \bar{z}^{n-1}
\end{aligned}
$$

Eqs. (36)-(38) are the governing equations for the present problem, which contain a set of unknown coefficients $\alpha_{m}(m=1, \ldots, \infty), b_{n}$ and $c_{n}(n=1, \ldots, \infty)$ and $b_{01}, b_{02}, c_{01}, c_{02}$. It is difficult to solve the 
governing equations analytically. We use the boundary collocation method to reduce the governing equations to a system of linear algebraic equations for the unknown coefficients and solve the problem. The crack surface is discretized into $M$ elements and the nodal points are given by the following expression:

$$
t_{k}=c+a_{0} \cos \theta_{k} \quad \theta_{k}=\frac{k \pi}{(M+1)} \quad k=1,2, \ldots, M
$$

The $i$ th outer edge of the rectangular plate is divided regularly into $N_{i}(i=1,2,3,4)$ segments. When the algebraic equations are solved, the complex potentials and the stress components produced by the crack and the loading on the outer edges are known. According to the superposition principle, the stress fields of the rectangular plate can be obtained.

\subsection{Governing equations for Case II}

Case II is shown in Fig. 2 and the difference between Cases I and II is in the loading form. In Fig. 2, a homogeneous stress $\left(\sigma_{x}^{0}\right)_{1}=\left(\sigma_{x}^{0}\right)_{2}=\sigma$ is loaded on the left and right edges, on the upper edge and lower edges is the homogeneous stress $\sigma_{y}^{0}$, where Eq. (28) is still satisfied. Also, the crack face is traction-free. The governing equation on the crack face for Case II is

$$
\begin{aligned}
& \left.\frac{\mu_{2}}{\kappa_{2}+1}\left\{2 \overline{I^{+}(t)}+2 t \overline{I^{++}(t)}-I^{\prime-}(t)\right]+I^{+}(t)-I^{-}(t)+\left(3 \Lambda_{2}-\Lambda_{1}\right) I(-t)-12 \Lambda_{2} t I^{\prime}(-t)+4 t^{2} \Lambda_{2} I^{\prime \prime}(-t)\right\} \\
& +\sum_{n=1}^{\infty} n\left(b_{n}+\lambda_{2} c_{n}\right) z^{n-1}+2 \sum_{n=1}^{\infty} n\left(\bar{b}_{n}+\lambda_{2} \bar{c}_{n}\right) \bar{z}^{n-1}-\lambda_{1} \sum_{n=1}^{\infty} n c_{n} \bar{z}^{n-1} \\
& \quad-\Lambda_{1} \sum_{n=1}^{\infty} n b_{n} \bar{z}^{n-1}+2 \sum_{n=1}^{\infty} n(n-1)\left(\bar{b}_{n}+\lambda_{2} \bar{c}_{n}\right) \bar{z}^{n-1}=0
\end{aligned}
$$

where $b<t<a$.

For Case II, we also use the resultant forces on each boundary as boundary conditions. Point $\mathrm{O}$ is assumed to be fixed at all times, a point $\mathrm{C}^{*}$ is permitted to move. The boundary conditions in the present analysis are written in terms of the resultant forces from $\mathrm{O}$ to $\mathrm{C}^{*}$ as follows:

$$
\begin{array}{ll}
\mathrm{C}^{*} \in \mathrm{OC}: & X+\mathrm{i} Y=\sigma y \\
\mathrm{C}^{*} \in \mathrm{CF}: & X+\mathrm{i} Y=-\sigma h_{2}+\mathrm{i} \sigma_{y}^{0}(w-x) \\
\mathrm{C}^{*} \in \mathrm{OD}: & X+\mathrm{i} Y=\sigma y \\
\mathrm{C}^{*} \in \mathrm{DE}: & X+\mathrm{i} Y=\sigma h_{1}+\mathrm{i} \sigma_{y}^{0}(w-x)
\end{array}
$$

Also the boundary collocation method is used to solve the governing equations for Case II.

\subsection{Stress intensity factor}

The stress distribution ahead of the crack tip B can be expressed as follows:

$$
\begin{aligned}
\sigma_{x}= & \frac{\mu_{2}}{\kappa_{2}+1}\left[2 I(t)+\left(3 \Lambda_{2}-\Lambda_{1}\right) I(-t)-12 \Lambda_{2} t I^{\prime}(-t)+4 \Lambda_{2} t^{2} I^{\prime \prime}(-t)\right]+\sum_{n=1}^{\infty} n\left(b_{n}+\lambda_{2} c_{n}\right) z^{n-1} \\
& +2 \sum_{n=1}^{\infty} n\left(\bar{b}_{n}+\lambda_{2} \bar{c}_{n}\right) \bar{z}^{n-1}-\lambda_{1} \sum_{n=1}^{\infty} n c_{n} \bar{z}^{n-1}-\Lambda_{1} \sum_{n=1}^{\infty} n b_{n} \bar{z}^{n-1}+2 \sum_{n=1}^{\infty} n(n-1)\left(\bar{b}_{n}+\lambda_{2} \bar{c}_{n}\right) \bar{z}^{n-1}
\end{aligned}
$$




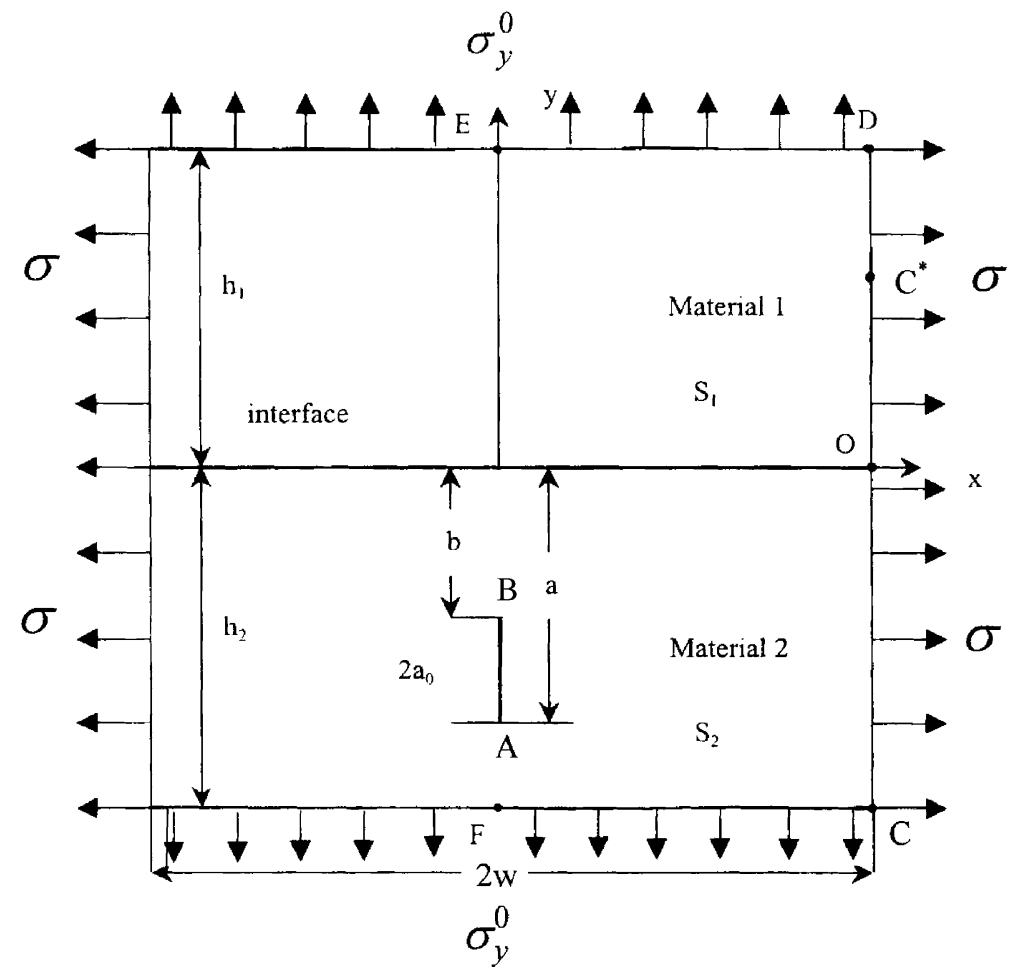

Fig. 2. A finite crack perpendicular to the bimaterial interface of a finite solid for case II.

and

$$
\begin{aligned}
\sigma_{y}= & \frac{\mu_{2}}{\kappa_{2}+1}\left[2 I(t)+\left(\Lambda_{2}+\Lambda_{1}\right) I(-t)+4 \Lambda_{2} t I^{\prime}(-t)-4 \Lambda_{2} t^{2} I^{\prime \prime}(-t)\right]+\sum_{n=1}^{\infty} n\left(3 b_{n}+3 \lambda_{2} c_{n}\right) z^{n-1} \\
& -2 \sum_{n=1}^{\infty} n\left(\bar{b}_{n}+\lambda_{2} \bar{c}_{n}\right) \bar{z}^{n-1}+\lambda_{1} \sum_{n=1}^{\infty} n c_{n} \bar{z}^{n-1}+\Lambda_{1} \sum_{n=1}^{\infty} n b_{n} \bar{z}^{n-1}-2 \sum_{n=1}^{\infty} n(n-1)\left(\bar{b}_{n}+\lambda_{2} \bar{c}_{n}\right) \bar{z}^{n-1}
\end{aligned}
$$

The stress intensity factor of crack tip B can be obtained as follows:

$$
K_{1(\mathrm{~B})}=\lim _{r \rightarrow 0} \sqrt{2 \pi r} \sigma_{x}=-\frac{2 \mu_{2}}{\kappa_{2}+1} \sqrt{\pi a_{0}} \sum_{m=1}^{\infty}(-1)^{m} \alpha_{m}
$$

The stress intensity factor of crack tip A is

$$
K_{1(\mathrm{~A})}=\frac{2 \mu_{2}}{\kappa_{2}+1} \sqrt{\pi a_{0}} \sum_{m=1}^{\infty} \alpha_{m}
$$

\section{6. $T$ stress}

The $T$ stress is the second term of the Williams series. Linear elastic fracture mechanics is usually based on the assumption that the stress fields near the crack tip are the $K$ field. However, much work has shown that single stress intensity might not suffice to characterize the crack tip fields. $T_{x}$ is the $x$-component of the $T$ stress contributed by the solution. For Case I, the total $T_{x}$ stress can be obtained as follows: 


$$
\begin{aligned}
T_{x}= & \frac{\mu_{2}}{\kappa_{2}+1}\left[2 \sum_{m=1}^{\infty}(-1)^{m} m \alpha_{m}+\left(3 \Lambda_{2}-\Lambda_{1}\right) I(-b)-12 \Lambda_{2} b I^{\prime}(-b)+4 \Lambda_{2} b^{2} I^{\prime \prime}(-b)\right] \\
& +\left(3-\Lambda_{1}\right) b_{1}+\left(3 \lambda_{2}-\lambda_{1}\right) c_{1}+\sigma
\end{aligned}
$$

For Case II, the total $T_{x}$ stress is

$$
\begin{aligned}
T_{x}= & \frac{\mu_{2}}{\kappa_{2}+1}\left[2 \sum_{m=1}^{\infty}(-1)^{m} m \alpha_{m}+\left(3 \Lambda_{2}-\Lambda_{1}\right) I(-b)-12 \Lambda_{2} b I^{\prime}(-b)+4 \Lambda_{2} b^{2} I^{\prime \prime}(-b)\right] \\
& +\left(3-\Lambda_{1}\right) b_{1}+\left(3 \lambda_{2}-\lambda_{1}\right) c_{1}
\end{aligned}
$$

It should be noted that the total $T_{x}$ stresses for both Cases I and II are zero since the crack face is tractionfree, which is proved also by the numerical calculation. This result also explains the final results in the present paper that $K$ field can describe the stress distribution in $x$-axis direction.

On the other hand, we have

$$
\sigma_{x}+\sigma_{y}=4 \operatorname{Re}\{\Phi(z)\}=\frac{4 \mu_{2}}{\kappa_{2}+1}\left\{I(t)+\Lambda_{2}\left[I(-t)-2 t I^{\prime}(-t)\right]\right\}+4 \lambda_{2} \operatorname{Re}\{G(z)\}+4 \operatorname{Re}\{F(z)\}
$$

A similar analysis shows that,

$$
T_{x}+T_{y}=\frac{4 \mu_{2}}{\kappa_{2}+1}\left\{\sum_{m=1}^{\infty}(-1)^{m} m \alpha_{m}+\Lambda_{2}\left[I(-b)-2 b I^{\prime}(-b)\right]\right\}+4 \lambda_{2} c_{1}+4 b_{1}
$$

Then, we can obtain the following equations for Cases I and II, respectively,

$$
\begin{aligned}
T_{y}= & \frac{\mu_{2}}{\kappa_{2}+1}\left[2 \sum_{m=1}^{\infty}(-1)^{m} m \alpha_{m}+\left(\Lambda_{2}+\Lambda_{1}\right) I(-b)+4 \Lambda_{2} b I^{\prime}(-b)-4 \Lambda_{2} b^{2} I^{\prime \prime}(-b)\right] \\
& +\left(1+\Lambda_{1}\right) b_{1}+\left(\lambda_{2}+\lambda_{1}\right) c_{1}-\sigma \\
T_{y}= & \frac{\mu_{2}}{\kappa_{2}+1}\left[2 \sum_{m=1}^{\infty}(-1)^{m} m \alpha_{m}+\left(\Lambda_{2}+\Lambda_{1}\right) I(-b)+4 \Lambda_{2} b I^{\prime}(-b)-4 \Lambda_{2} b^{2} I^{\prime \prime}(-b)\right] \\
& +\left(1+\Lambda_{1}\right) b_{1}+\left(\lambda_{2}+\lambda_{1}\right) c_{1}
\end{aligned}
$$

\section{Calculation results}

\subsection{Infinite solid problem}

In order to verify our program, we calculate an infinite solid problem first with $b_{n}=c_{n}=0$. Typical examples for aluminum-epoxy and epoxy-boron in the case of $b / a_{0}=0.01$ and $b / a_{0}=0.1$ were tested. The coefficients $\alpha_{m}$ approach to zero rapidly as $m$ increases. All the results given for this problem were calculated with $M=100$.

Figs. 3 and 4 show the stress distribution ahead of the crack tip B for aluminum-epoxy in the case of $b / a_{0}=0.01$, which means that the crack lies in the weaker material. It is clear that the normal stress $\sigma_{x}$ is dominated by the $K$ field in the region of $0<r / b<0.5$, meanwhile the normal stress $\sigma_{y}$ is influenced by both the $K$ field and the $T$ stress and the single $K$ field cannot adequately describe the stress component in $y$ direction. It is clear that the $T$ stress effect is very important for aluminum-epoxy. 


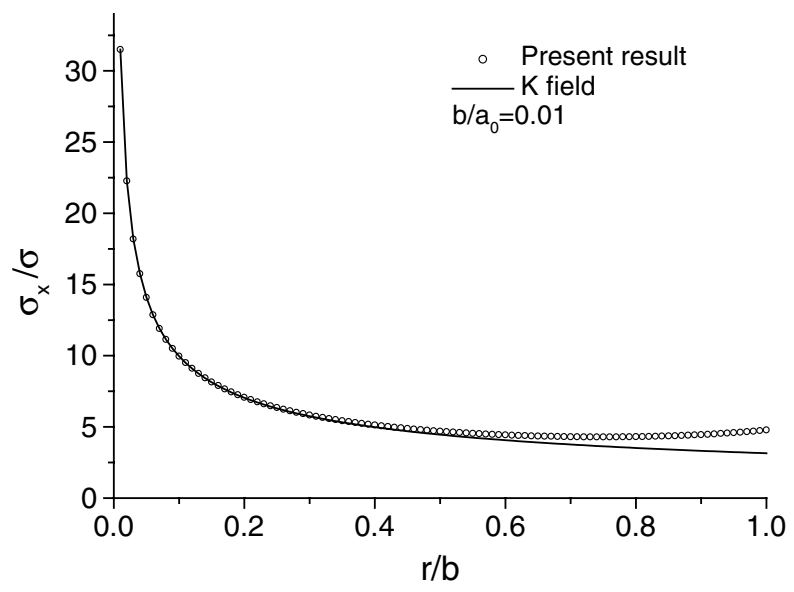

Fig. 3. Normalized stress $\sigma_{x}$ distribution ahead of the crack tip B versus $r / b$ for an infinite aluminum-epoxy bimaterial plate with $b / a_{0}=0.01$.

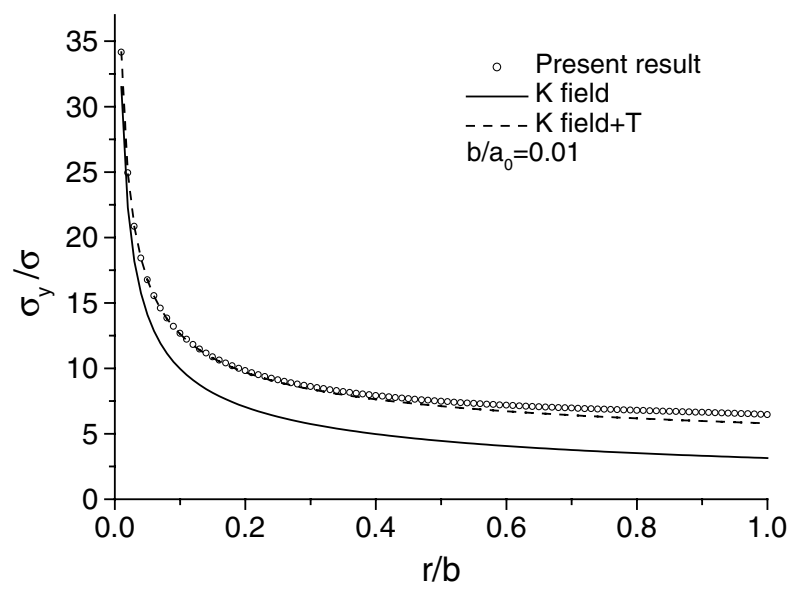

Fig. 4. Normalized stress $\sigma_{y}$ distribution ahead of the crack tip B versus $r / b$ for an infinite aluminum-epoxy bimaterial plate with $b / a_{0}=0.01$.

Figs. 5 and 6 show the stress distribution ahead of the crack tip B for epoxy-boron in the case of $b / a_{0}=0.1$. Also, one can find that the normal stress $\sigma_{x}$ is dominated by the $K$ field and $\sigma_{y}$ is influenced by both the $K$ field and the $T$ stress, in the region of $0<r / b<0.5$. In this case the crack lies in the stiffer material. All the above calculation results for an infinite solid are consistent with those given by Wang and Ståhle $(1998 \mathrm{a}, \mathrm{b})$.

\subsection{Finite solid problem}

What is investigated mainly in the present paper is a crack perpendicular to the interface in a finite solid, where the influence of outer boundaries must be considered. The following results are given for Case I as shown in Fig. 1 and Case II as shown in Fig. 2, respectively. 


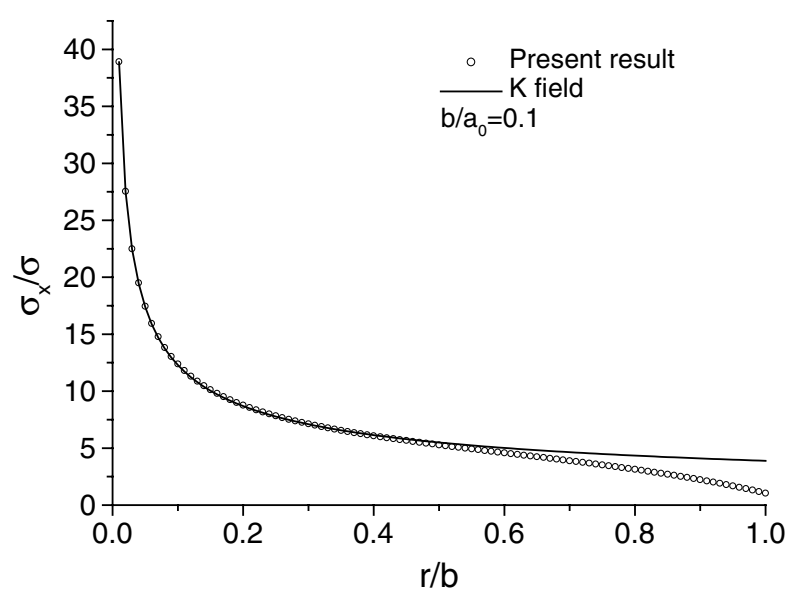

Fig. 5. Normalized stress $\sigma_{x}$ distribution ahead of the crack tip B versus $r / b$ for an infinite epoxy-boron bimaterial plate with $b / a_{0}=0.1$.

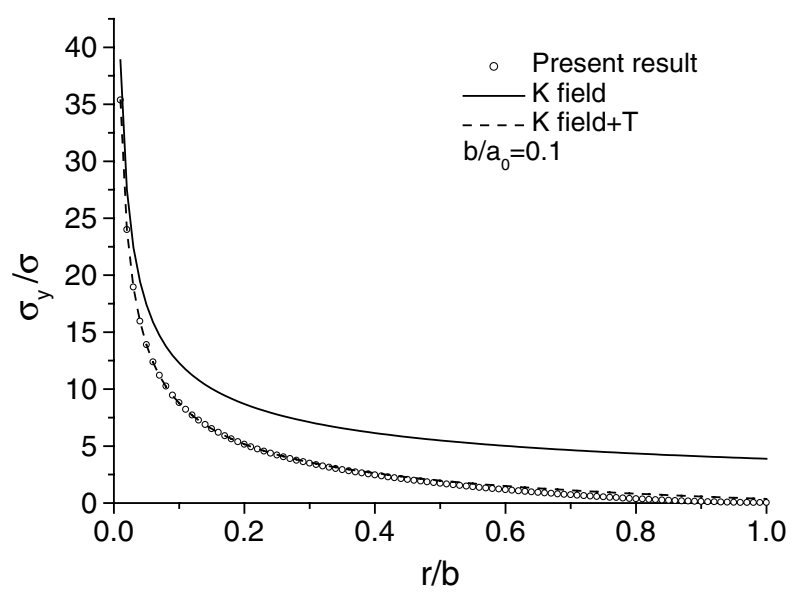

Fig. 6. Normalized stress $\sigma_{y}$ distribution ahead of the crack tip B versus $r / b$ for an infinite epoxy-boron bimaterial plate with $b / a_{0}=0.1$.

\subsubsection{The results for Case I}

Figs. 7 and 8 show the stress distribution ahead of the crack tip B versus the normalized distance, $r / b$, for aluminum-epoxy. Poisson's ratio of aluminum is $v_{1}=0.3$ and that of epoxy is $v_{2}=0.35$. Shear modulus ratio of the two materials is $\mu_{1} / \mu_{2}=23.08$, which means that the crack is in a weaker material. The normalized parameters are $w / a_{0}=5.0, h_{1} / a_{0}=h_{2} / a_{0}=5.0$. The normalized distance between the crack tip B and the interface is $b / a_{0}=0.01$. From Fig. 7, we see that the $K$ field can characterize the stress field in $x$ direction very well in the region of $0<r / b<0.5$. Fig. 8 shows that the $K$ field does not describe adequately the stress field in $y$-direction and both the $K$ field and the $T$ stress should be used to be consistent well with the present result in the region of $0<r / b<0.5$. Comparing Figs. 7 and 8 with Figs. 3 and 4, one can find that the $K$ field is consistent with the stress field in $x$-direction and the $K$ field plus the $T$ stress can describe the stress field in $y$-direction either for an infinite problem or for a finite problem. 


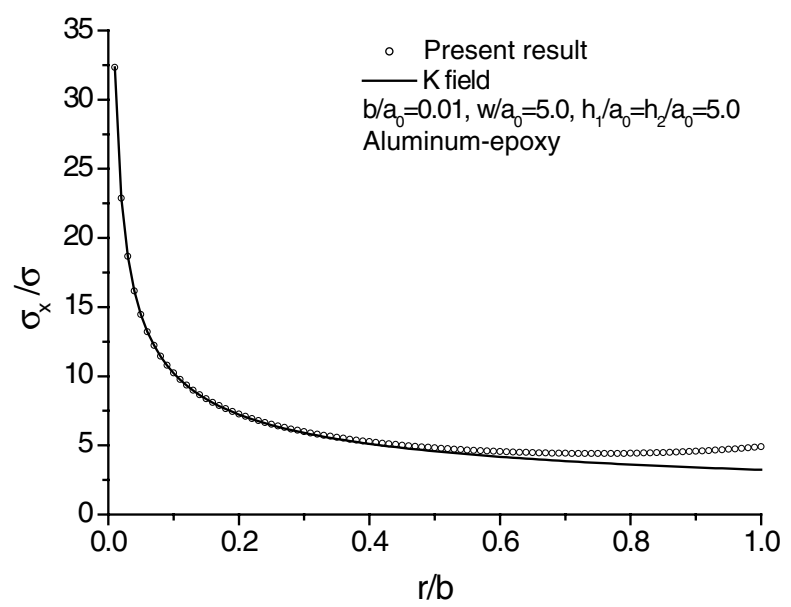

Fig. 7. Normalized stress $\sigma_{x}$ distribution ahead of the crack tip B versus $r / b$ for a finite aluminum-epoxy bimaterial plate with $b / a_{0}=0.01$.

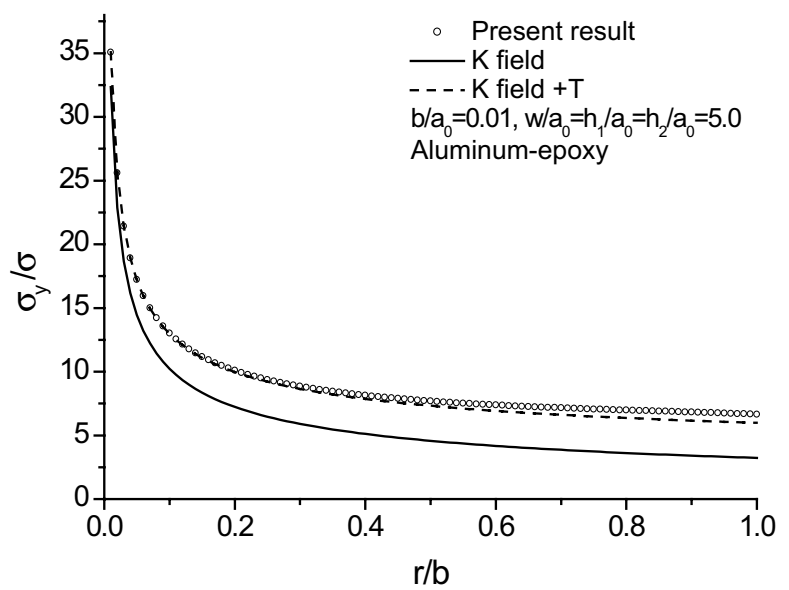

Fig. 8. Normalized stress $\sigma_{y}$ distribution ahead of the crack tip B versus $r / b$ for a finite aluminum-epoxy bimaterial plate with $b / a_{0}=0.01$.

Figs. 9 and 10 show the stress distribution ahead of the crack tip B for $b / a_{0}=1.0$ with other parameters being the same as those in Figs. 7 and 8. From Figs. 9 and 10, we also see that the $K$ field is consistent with the stress field in $x$-direction and both the $K$ field and the $T$ stress should be used for the stress field in $y$ direction in the region of $0<r / b<0.1$. Comparing Figs. 9 and 10 with Figs. 7 and 8 , one can see that the distance $b$ has a significant influence on the stress intensity factor so that the normalized stresses are quite different in Figs. 7 and 8 and in Figs. 9 and 10. When the crack lies in a weaker material, the stress intensity factor decreases with increasing $b$. Comparing Figs. 8 and 10, we can see that the $T$ stress in $y$-direction will become positive and tensile when the distance of crack tip B to the interface decreases, which means that the crack path is unstable and will change its advancing direction when the crack lies in a weaker material and the crack tip is near the interface. This result is consistent with that given by Ye et al. (1992). 


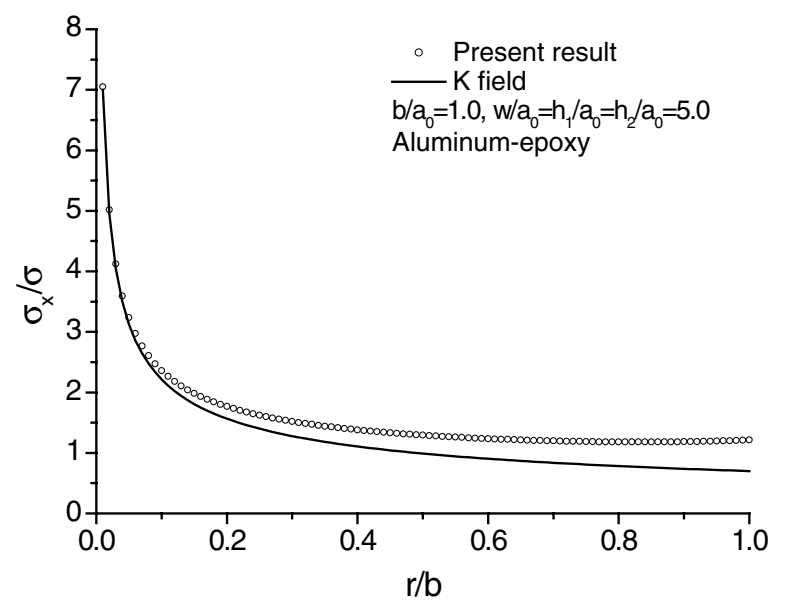

Fig. 9. Normalized stress $\sigma_{x}$ distribution ahead of the crack tip B versus $r / b$ for a finite aluminum-epoxy bimaterial plate with $b / a_{0}=1.0$.

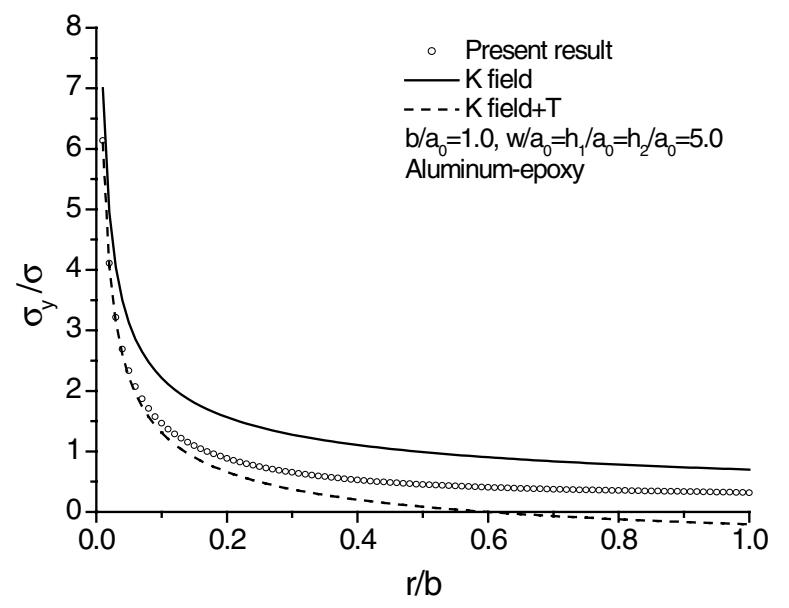

Fig. 10. Normalized stress $\sigma_{y}$ distribution ahead of the crack tip B versus $r / b$ for a finite aluminum-epoxy bimaterial plate with $b / a_{0}=1.0$.

When the crack lies in a stiffer material, the thing may be different from the case when the crack lies in a weaker material. We give the corresponding normalized stress distributions versus the normalized distance from crack tip B, $r / b$, for the same edge length as those used in Figs. 9 and 10, but with different shear ratio. Figs. 11 and 12 show the normalized stress distribution for epoxy-boron and the shear ratio is $\mu_{1} / \mu_{2}=0.007223$. Poisson's ratio of epoxy is $v_{1}=0.3$ and that of boron is $v_{2}=0.35$ and the normalized distance $b$ from the crack tip B to the interface is $b / a_{0}=0.1$. Figs. 13 and 14 show the normalized stress distribution ahead of the crack tip $\mathrm{B}$ versus the normalized distance from the crack tip $\mathrm{B}, r / b$, for the case of $b / a_{0}=1.0$ with other parameters being the same as those used in Figs. 11 and 12. The phenomena found in the case when the crack lies in a weaker material can also be observed in the case when the crack lies in a stiffer material. Comparing Figs. 12 and 14, we can see that the $T$ stress is always compressive stress when 


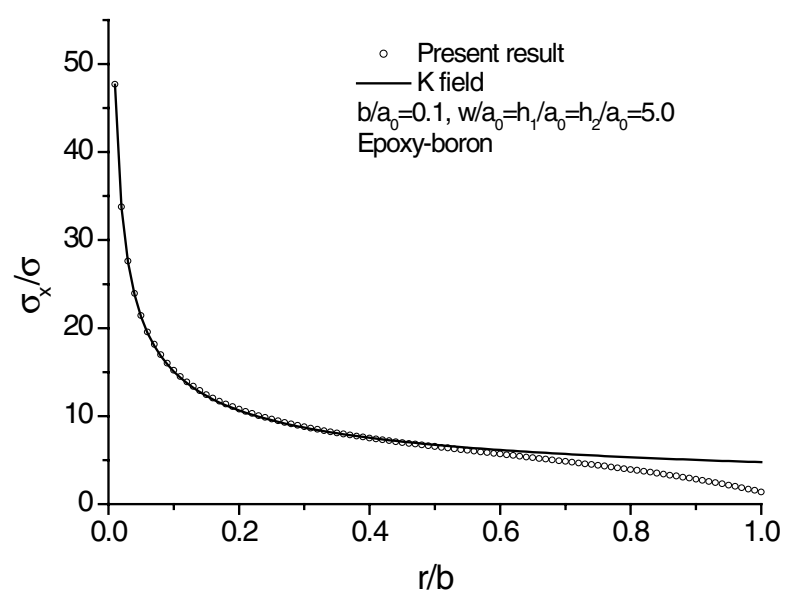

Fig. 11. Normalized stress $\sigma_{x}$ distribution ahead of the crack tip B versus $r / b$ for a finite epoxy-boron bimaterial plate with $b / a_{0}=0.1$.

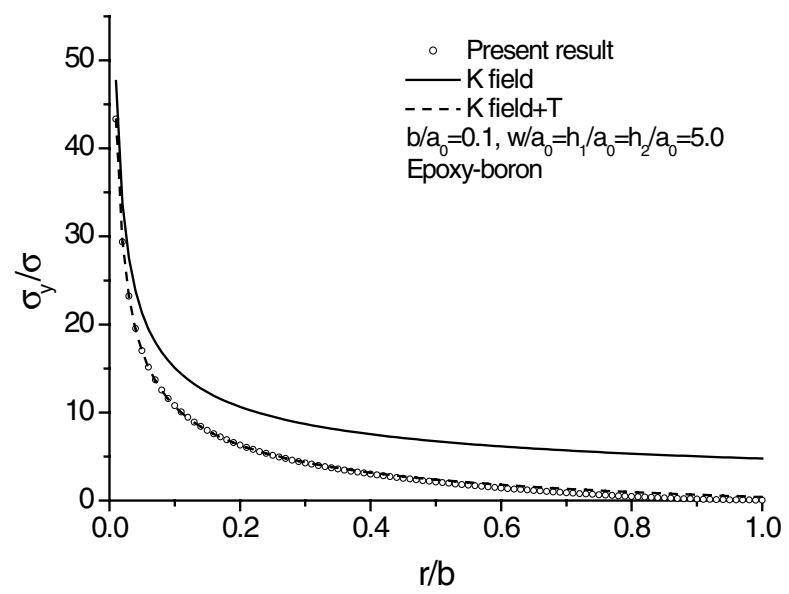

Fig. 12. Normalized stress $\sigma_{y}$ distribution ahead of the crack tip B versus $r / b$ for a finite epoxy-boron bimaterial plate with $b / a_{0}=0.1$.

the crack lies in a stiffer material, which means the crack path is always stable in that case. This result is consistent well with the experimental result given by Ståhle et al. (1995).

All the above results are related with the effect of the distance $b$ and the crack's position. For a finite problem, the scale of the sample must have an influence on the stress intensity factor. Now we consider the cases with different sample scales but with other parameters being the same.

Table 1 shows the normalized stress intensity factors $K_{2}(\mathrm{~B}) / \sigma \sqrt{\pi a_{0}}$ for different ratios of the scale of the sample to the crack length versus different shear ratios, while the crack lies in a weaker material 2 and $b / a_{0}=0.1, v_{1}=0.3$. In Table 1 , four kinds of samples with different scales are shown with different shear ratios, while the crack lengths are the same. From Table 1, one can see that the stress intensity factor will increase when the size of the sample decreases for the same shear ratio. The stress intensity factor is the smallest for the infinite problems. The stress intensity factor will decrease when the shear ratio of the upper material to the lower material increases for the same sample scale. 


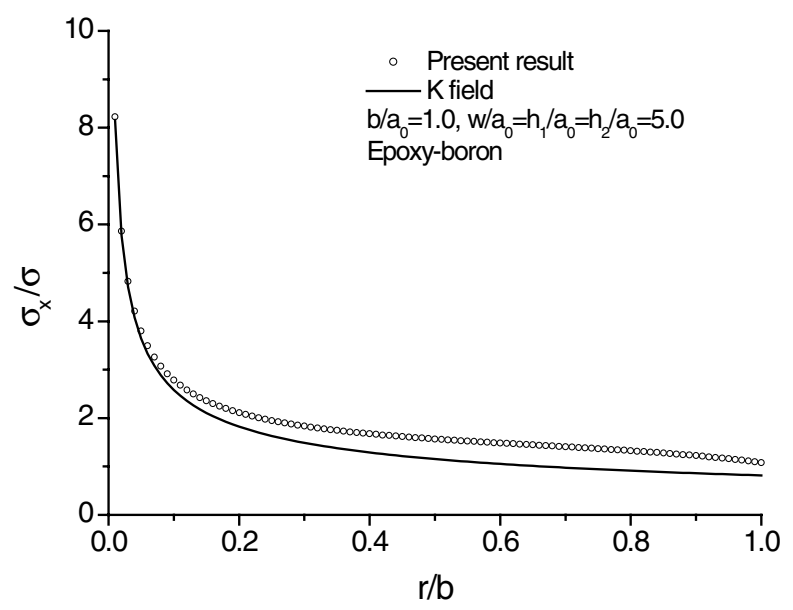

Fig. 13. Normalized stress $\sigma_{x}$ distribution ahead of the crack tip B versus $r / b$ for a finite epoxy-boron bimaterial plate with $b / a_{0}=1.0$.

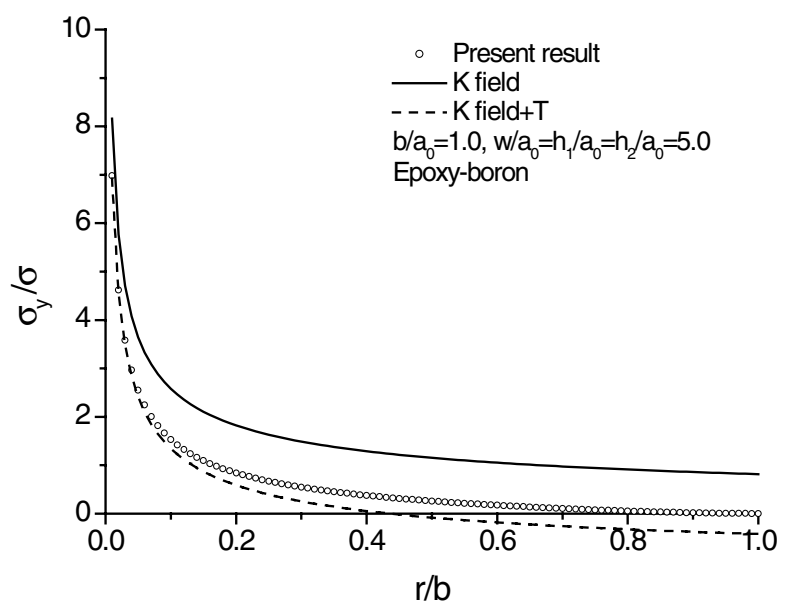

Fig. 14. Normalized stress $\sigma_{y}$ distribution ahead of the crack tip B versus $r / b$ for a finite epoxy-boron bimaterial plate with $b / a_{0}=1.0$.

Table 2 shows the normalized stress intensity factor $K_{2}(\mathrm{~B}) / \sigma \sqrt{\pi a_{0}}$ for different ratios of the scale of the sample to the crack length versus different shear ratios, while the crack lies in a stiffer material 2 and $b / a_{0}=0.1, v_{1}=0.35, v_{2}=0.3$. In Table 2 , four kinds of samples with different scales are shown with different shear ratios, while the crack lengths are the same. From Table 2, one can also see that the stress intensity factor will increase when the size of the sample decreases for the same shear ratio. The stress intensity factor is the smallest for the infinite problems. The stress intensity factor will increase when the shear ratio of the lower material to the upper material increases for the same sample scale.

From Tables 1 and 2, one can find also that the normalized stress intensity factors are smaller than those for the homogeneous material, when the crack lies in a weaker material; they are larger than those for the homogeneous material, when the crack lies in a stiffer material. As for the case of an infinite solid with $\mu_{1} / \mu_{2}=1.0$ in Tables 1 and 2, we can see the influence of Poisson's ratio. If $v_{1} / v_{2}=1.0$, the normalized stress intensity factor is unity. 
Table 1

$K_{2}(\mathrm{~B}) / \sigma \sqrt{\pi a_{0}}$ for sample scales with the same crack length, when the crack lies in a weaker material $\left(b / a_{0}=0.1, v_{1}=0.3, v_{2}=0.35\right)$

\begin{tabular}{|c|c|c|c|c|c|c|c|c|c|}
\hline & \multicolumn{9}{|l|}{$\underline{\mu_{1} / \mu_{2}}$} \\
\hline & 1000.0 & 800.0 & 500.0 & 250.0 & 100.0 & 50.0 & 10.0 & 5.0 & 1.0 \\
\hline $\begin{array}{l}w / a_{0}=3 \\
h_{1} / a_{0}=3 \\
h_{2} / a_{0}=3\end{array}$ & 0.700 & 0.701 & 0.703 & 0.707 & 0.715 & 0.725 & 0.788 & 0.924 & 1.181 \\
\hline $\begin{array}{l}w / a_{0}=5 \\
h_{1} / a_{0}=5 \\
h_{2} / a_{0}=5\end{array}$ & 0.6670 & 0.6671 & 0.6672 & 0.6677 & 0.669 & 0.673 & 0.718 & 0.775 & 1.070 \\
\hline $\begin{array}{l}w / a_{0}=10 \\
h_{1} / a_{0}=10 \\
h_{2} / a_{0}=10\end{array}$ & 0.6440 & 0.6442 & 0.6446 & 0.6458 & 0.649 & 0.655 & 0.703 & 0.756 & 1.028 \\
\hline Infinite solid & 0.6407 & 0.6408 & 0.6413 & 0.6426 & 0.6465 & 0.6529 & 0.7001 & 0.7517 & 1.014 \\
\hline
\end{tabular}

Table 2

$K_{2}(B) / \sigma \sqrt{\pi a_{0}}$ for sample scales with the same crack length, when the crack lies in a stiffer material $\left(b / a_{0}=0.1, v_{1}=0.35, v_{2}=0.3\right)$

\begin{tabular}{|c|c|c|c|c|c|c|c|c|c|}
\hline & \multicolumn{9}{|l|}{$\mu_{1} / \mu_{2}$} \\
\hline & 1000.0 & 800.0 & 500.0 & 250.0 & 100.0 & 50.0 & 10.0 & 5.0 & 1.0 \\
\hline $\begin{array}{l}w / a_{0}=3 \\
h_{1} / a_{0}=3 \\
h_{2} / a_{0}=3\end{array}$ & 2.408 & 2.399 & 2.374 & 2.326 & 2.247 & 2.172 & 1.867 & 1.654 & 1.140 \\
\hline $\begin{array}{l}w / a_{0}=5 \\
h_{1} / a_{0}=5 \\
h_{2} / a_{0}=5\end{array}$ & 2.288 & 2.276 & 2.246 & 2.188 & 2.096 & 2.016 & 1.721 & 1.519 & 1.039 \\
\hline $\begin{array}{l}w / a_{0}=10 \\
h_{1} / a_{0}=10 \\
h_{2} / a_{0}=10\end{array}$ & 1.935 & 1.932 & 1.922 & 1.901 & 1.862 & 1.819 & 1.602 & 1.435 & 1.0 \\
\hline Infinite solid & 1.755 & 1.754 & 1.752 & 1.747 & 1.730 & 1.704 & 1.536 & 1.393 & 0.985 \\
\hline
\end{tabular}

Figs. 15 and 16 give the normalized stress intensity factor versus the shear modulus ratio $\mu_{1} / \mu_{2}$ with different normalized distances from the crack tip B to the interface for two cases, when the crack lies in a weaker material and when the crack lies in a stiffer material. In Fig. 15, it is shown that when the normalized distance $b / a_{0}$ decreases, the stress intensity factor will decrease also. Fig. 16 shows that when the normalized distance $b / a_{0}$ decreases, the stress intensity factor will increase.

\subsubsection{The results for Case II}

Figs. 17 and 18 show the stress distribution ahead of the crack tip B versus the normalized distance, $r / b$, for aluminum-epoxy. Poisson's ratio of aluminum is $v_{1}=0.3$ and that of epoxy is $v_{2}=0.35$. The shear ratio of the two materials is $\mu_{1} / \mu_{2}=23.08$, which means the crack is in a weaker material. The normalized parameters are $w / a_{0}=5.0, h_{1} / a_{0}=h_{2} / a_{0}=5.0$. The normalized distance between the crack tip B and the interface is $b / a_{0}=0.01$. From Fig. 17, we can see that the $K$ field can characterize the stress field in $x$ direction very well in the region of $0<r / b<0.5$. Fig. 18 shows that the $K$ field cannot describe adequately the stress field in $y$-direction and both the $K$ field and the $T$ stress should be considered to be consistent well with the present result in the region of $0<r / b<0.5$. Comparing Figs. 17 and 18 with Figs. 3 and 4 , one 


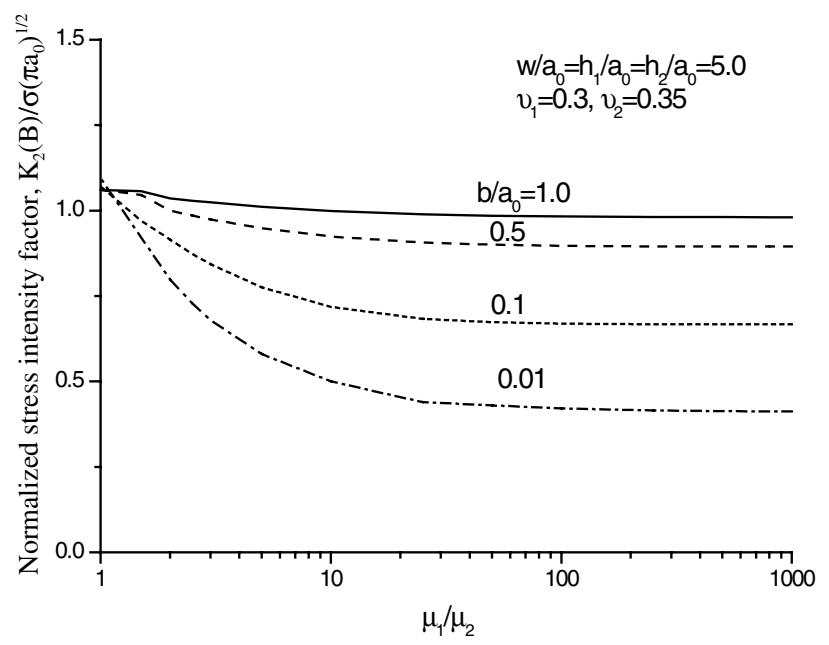

Fig. 15. Normalized stress intensity $K_{2}(\mathrm{~B}) / \sigma \sqrt{\pi a_{0}}$ versus $\mu_{1} / \mu_{2}$ for a finite bimaterial solid with different ratio of $b / a_{0}$, when the crack lies in a weaker material.

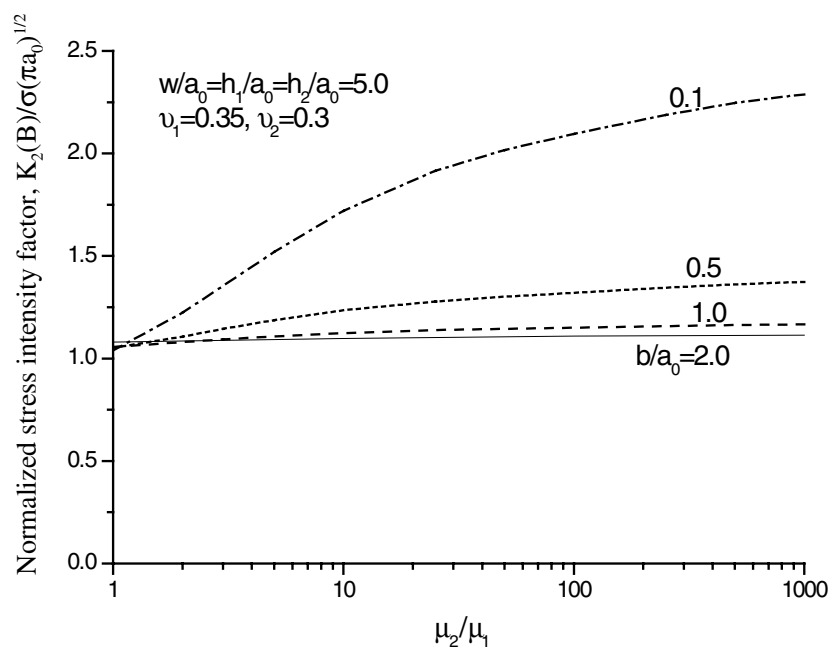

Fig. 16. Normalized stress intensity $K_{2}(\mathrm{~B}) / \sigma \sqrt{\pi a_{0}}$ versus $\mu_{2} / \mu_{1}$ for a finite bimaterial solid with different ratio of $b / a_{0}$, when the crack lies in a stiffer material.

can see that the $K$ field is consistent with the stress field in $x$-direction and the $K$ field plus the $T$ stress can describe the stress field in $y$-direction either for an infinite problem or for a finite problem. Comparing Cases I and II, one can see that Fig. 17 is the same as Fig. 7.

Figs. 19 and 20 show the stress distribution ahead of the crack tip B for $b / a_{0}=1.0$ with other parameters being the same as those in Figs. 7 and 8. From Figs. 19 and 20, we can also see that the $K$ field is consistent with the stress field in $x$-direction and the $K$ field plus the $T$ stress characterize the stress field in $y$-direction, in the region of $0<r / b<0.1$. Comparing Figs. 19 and 20 with Figs. 17 and 18, one can see that the distance $b$ has a significant influence on the stress intensity factor so that the normalized stresses are different in Figs. 17 and 18 and in Figs. 19 and 20, when the crack lies in a weaker material, the intensity decreases with increasing $b$. 


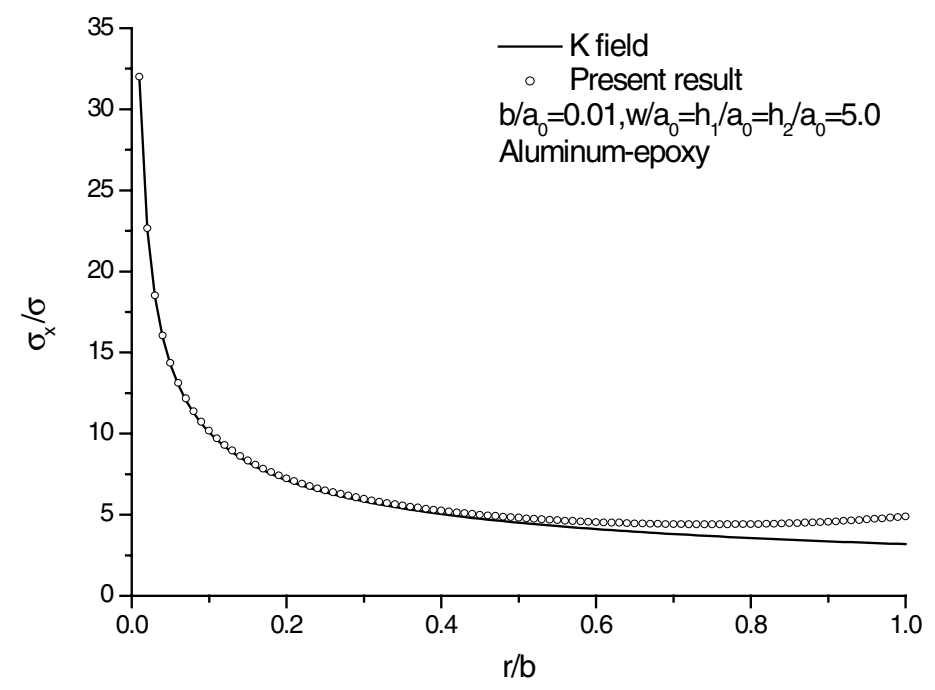

Fig. 17. Normalized stress $\sigma_{x}$ distribution ahead of the crack tip B versus $r / b$ for a finite aluminum-epoxy bimaterial plate with $b / a_{0}=0.01$.

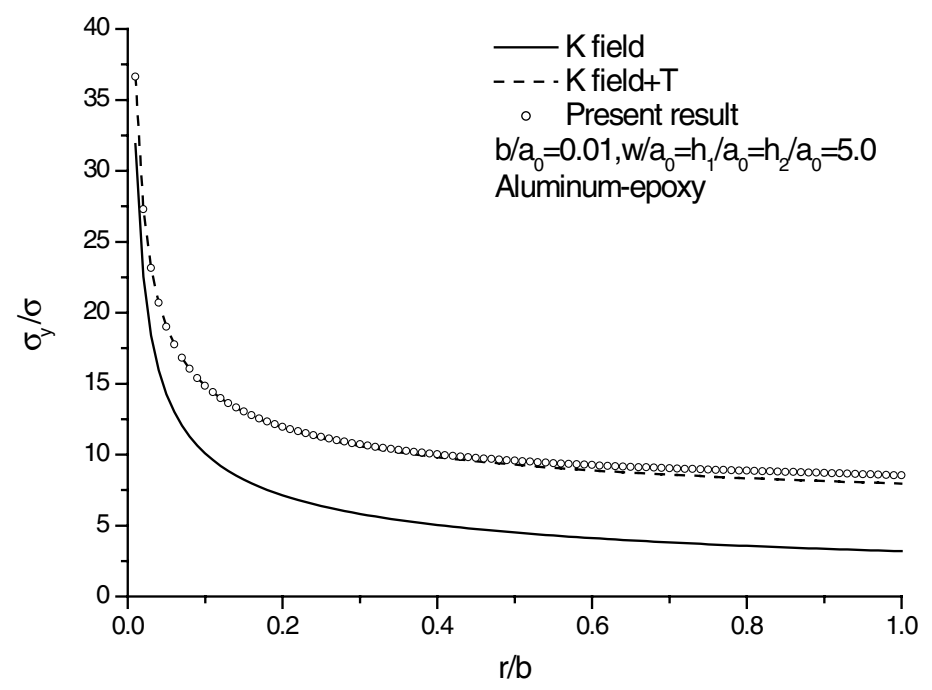

Fig. 18. Normalized stress $\sigma_{y}$ distribution ahead of the crack tip B versus $r / b$ for a finite aluminum-epoxy bimaterial plate with $b / a_{0}=0.01$.

When the crack lies in a stiffer material, the thing may be different from the case when the crack lies in the weaker material. We give the corresponding normalized stress distributions versus the normalized distance from crack tip B, $r / b$, for the same sample scales as those used in Figs. 19 and 20, but with different shear ratio. Figs. 21 and 22 show the normalized stress distribution for epoxy-boron and the shear ratio is $\mu_{1} / \mu_{2}=0.007223$. Poisson's ratio of epoxy is $v_{1}=0.3$, that of boron is $v_{2}=0.35$ and the normalized distance $b$ from the crack tip $\mathrm{B}$ to the interface is $b / a_{0}=0.1$. Figs. 23 and 24 show the normalized stress 


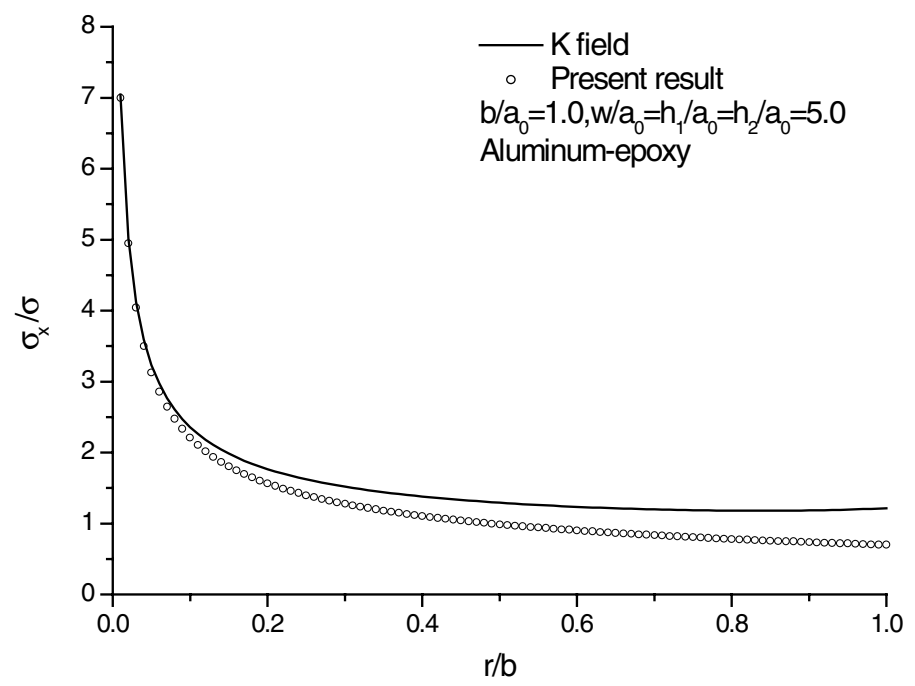

Fig. 19. Normalized stress $\sigma_{x}$ distribution ahead of the crack tip B versus $r / b$ for a finite aluminum-epoxy bimaterial plate with $b / a_{0}=1.0$.

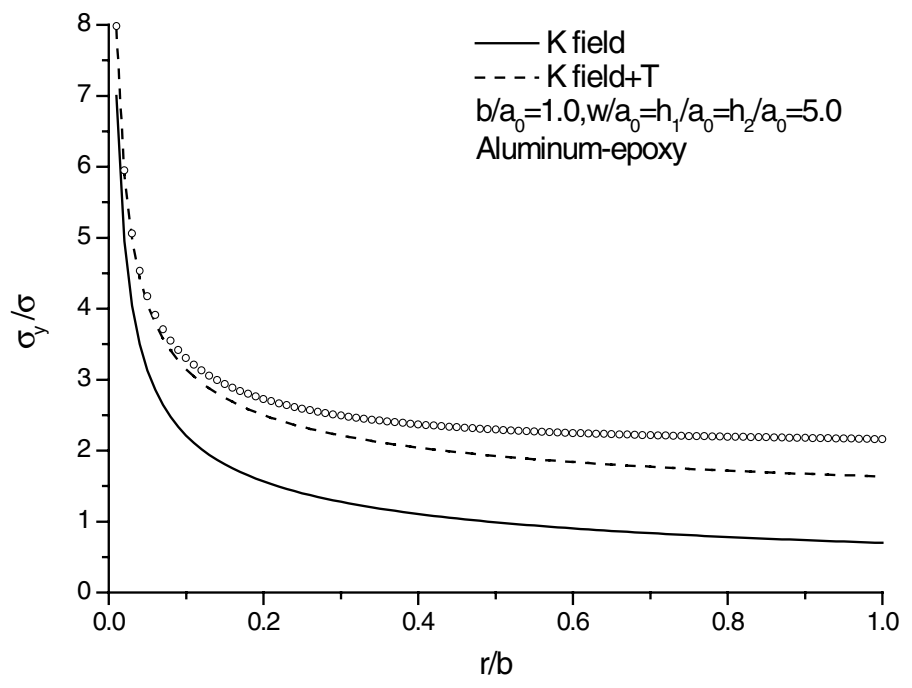

Fig. 20. Normalized stress $\sigma_{y}$ distribution ahead of the crack tip B versus $r / b$ for a finite aluminum-epoxy bimaterial plate with $b / a_{0}=1.0$.

distribution ahead of the crack tip B versus the normalized distance from the crack tip $\mathrm{B}, r / b$, for the case of $b / a_{0}=1.0$ and the other parameters are the same as those used in Figs. 21 and 22. The phenomena found in the case when the crack lies in a weaker material also can be observed in the case when the crack lies in a stiffer material.

Table 3 shows the normalized stress intensity factors $K_{2}(\mathrm{~B}) / \sigma \sqrt{\pi a_{0}}$ for different ratios of the scale of the sample to the crack length versus different shear ratios, when the crack lies in a weaker material and $b / a_{0}=0.1, v_{1}=0.3, v_{2}=0.35$. From Table 3, one can see that the stress intensity factor at crack tip point B 


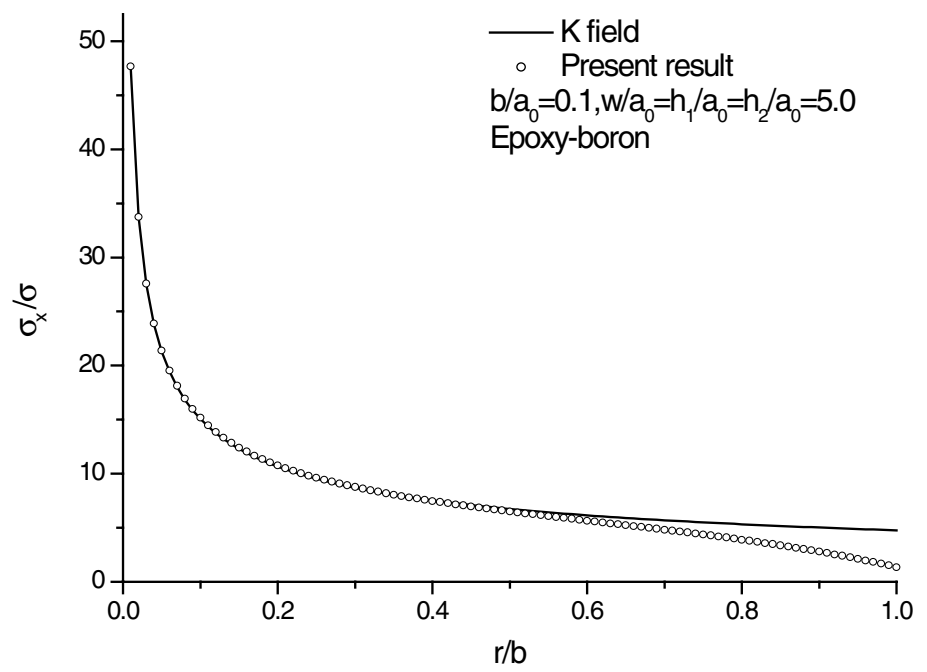

Fig. 21. Normalized stress $\sigma_{x}$ distribution ahead of the crack tip B versus $r / b$ for a finite epoxy-boron bimaterial plate with $b / a_{0}=0.1$.

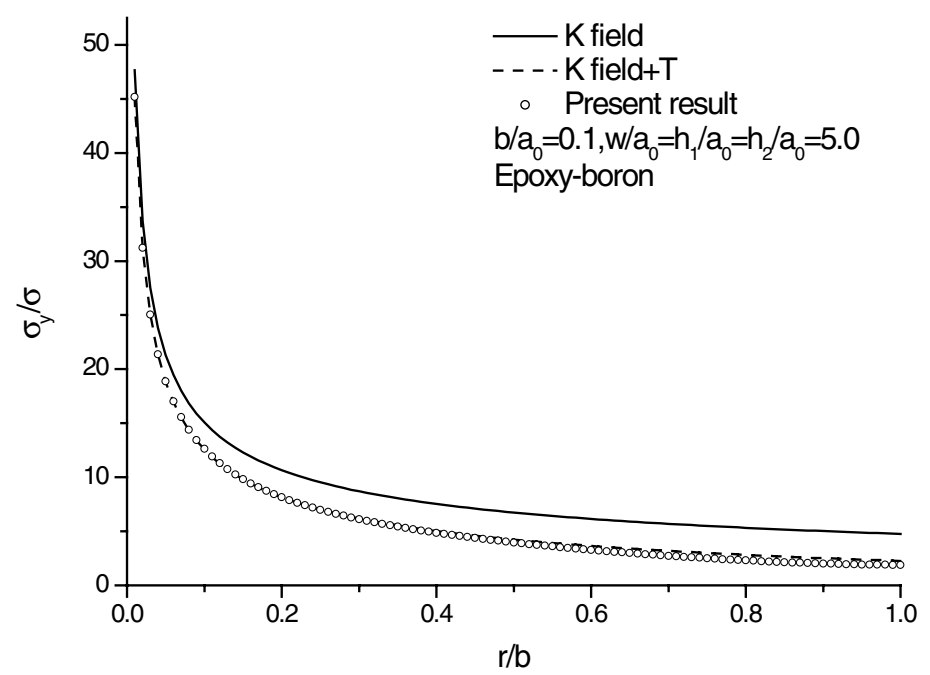

Fig. 22. Normalized stress $\sigma_{y}$ distribution ahead of the crack tip B versus $r / b$ for a finite epoxy-boron bimaterial plate with $b / a_{0}=0.1$.

will increase when the sample scale decreases for the same shear ratio. The stress intensity factor is the smallest for the infinite problems. The stress intensity factor at point B will decrease when the shear ratio of the upper material to the lower material increases for the same sample scale.

Table 4 shows the normalized stress intensity factor $K_{2}(\mathrm{~B}) / \sigma \sqrt{\pi a_{0}}$ for different ratios of the scale of the sample to the crack length versus different shear ratios, while the crack lies in a stiffer material and $b / a_{0}=0.1, v_{1}=0.35, v_{2}=0.3$. From Table 4 , one can also see that the stress intensity factor at the crack tip point $\mathrm{B}$ will increase when the ratio of the sample scale to the crack length decreases for the same shear ratio. The stress intensity factor is the smallest for the infinite problems. The stress intensity factor at point 


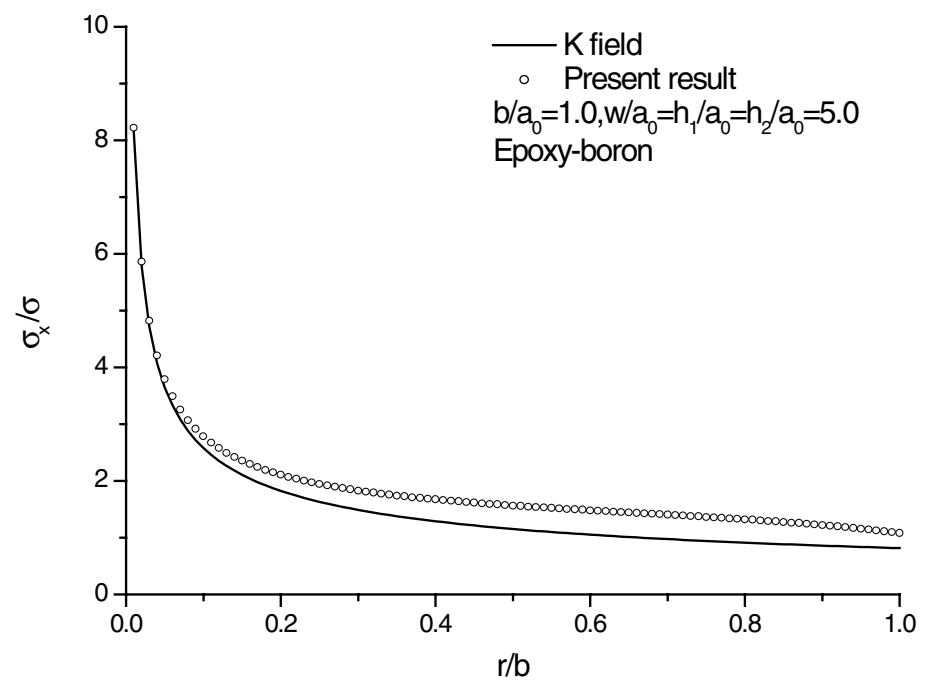

Fig. 23. Normalized stress $\sigma_{x}$ distribution ahead of the crack tip B versus $r / b$ for a finite epoxy-boron bimaterial plate with $b / a_{0}=1.0$.

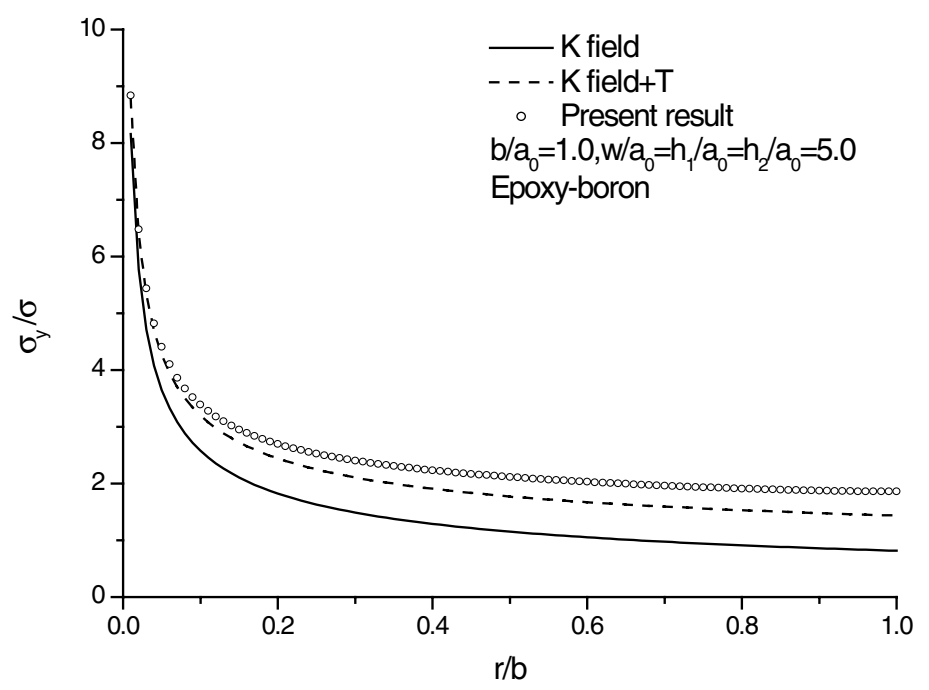

Fig. 24. Normalized stress $\sigma_{y}$ distribution ahead of the crack tip B versus $r / b$ for a finite epoxy-boron bimaterial plate with $b / a_{0}=1.0$.

B will increase when the shear ratio of the lower material to the upper material increases for the same sample scale.

From Tables 3 and 4, one can find also that the normalized stress intensity factors are smaller than those for the homogeneous material, when the crack lies in a weaker material; they are larger than those for the homogeneous material, when the crack lies in a stiffer material.

Figs. 25 and 26 give the normalized stress intensity factor versus the shear ratio $\mu_{1} / \mu_{2}$ with different normalized distances from the crack tip B to the interface for two cases, when the crack lies in a weaker material and when the crack lies in a stiffer material. In Fig. 25, when the normalized distance $b / a_{0}$ decreases, the stress intensity factor will decrease also. Fig. 26 shows that when the normalized distance $b / a_{0}$ decreases, the stress intensity factor will increase. 
Table 3

$K_{2}(B) / \sigma \sqrt{\pi a_{0}}$ for sample scales with the same crack length, when the crack lies in a weaker material $\left(b / a_{0}=0.1, v_{1}=0.3, v_{2}=0.35\right)$

\begin{tabular}{|c|c|c|c|c|c|c|c|c|c|}
\hline & \multicolumn{9}{|l|}{$\mu_{1} / \mu_{2}$} \\
\hline & 1000.0 & 800.0 & 500.0 & 250.0 & 100.0 & 50.0 & 10.0 & 5.0 & 1.0 \\
\hline $\begin{array}{l}w / a_{0}=3 \\
h_{1} / a_{0}=3 \\
h_{2} / a_{0}=3\end{array}$ & 0.702 & 0.703 & 0.705 & 0.709 & 0.717 & 0.727 & 0.790 & 0.854 & 1.181 \\
\hline $\begin{array}{l}w / a_{0}=5 \\
h_{1} / a_{0}=5 \\
h_{2} / a_{0}=5\end{array}$ & 0.6687 & 0.6688 & 0.6689 & 0.6693 & 0.671 & 0.675 & 0.720 & 0.777 & 1.070 \\
\hline $\begin{array}{l}w / a_{0}=10 \\
h_{1} / a_{0}=10 \\
h_{2} / a_{0}=10\end{array}$ & 0.6456 & 0.6458 & 0.6462 & 0.6474 & 0.651 & 0.657 & 0.704 & 0.758 & 1.028 \\
\hline
\end{tabular}

Table 4

$K_{2}(B) / \sigma \sqrt{\pi a_{0}}$ for sample scales with the same crack length, when the crack lies in a stiffer material $\left(b / a_{0}=0.1, v_{1}=0.35, v_{2}=0.3\right)$

\begin{tabular}{|c|c|c|c|c|c|c|c|c|c|}
\hline & \multicolumn{9}{|l|}{$\mu_{1} / \mu_{2}$} \\
\hline & 1000.0 & 800.0 & 500.0 & 250.0 & 100.0 & 50.0 & 10.0 & 5.0 & 1.0 \\
\hline $\begin{array}{l}w / a_{0}=3 \\
h_{1} / a_{0}=3 \\
h_{2} / a_{0}=3\end{array}$ & 2.604 & 2.602 & 2.593 & 2.571 & 2.512 & 2.426 & 2.002 & 1.727 & 1.138 \\
\hline $\begin{array}{l}w / a_{0}=5 \\
h_{1} / a_{0}=5 \\
h_{2} / a_{0}=5\end{array}$ & 2.343 & 2.341 & 2.333 & 2.314 & 2.262 & 2.187 & 1.818 & 1.574 & 1.039 \\
\hline $\begin{array}{l}w / a_{0}=10 \\
h_{1} / a_{0}=10 \\
h_{2} / a_{0}=10\end{array}$ & 1.955 & 1.954 & 1.950 & 1.940 & 1.912 & 1.870 & 1.633 & 1.453 & 1.0 \\
\hline
\end{tabular}

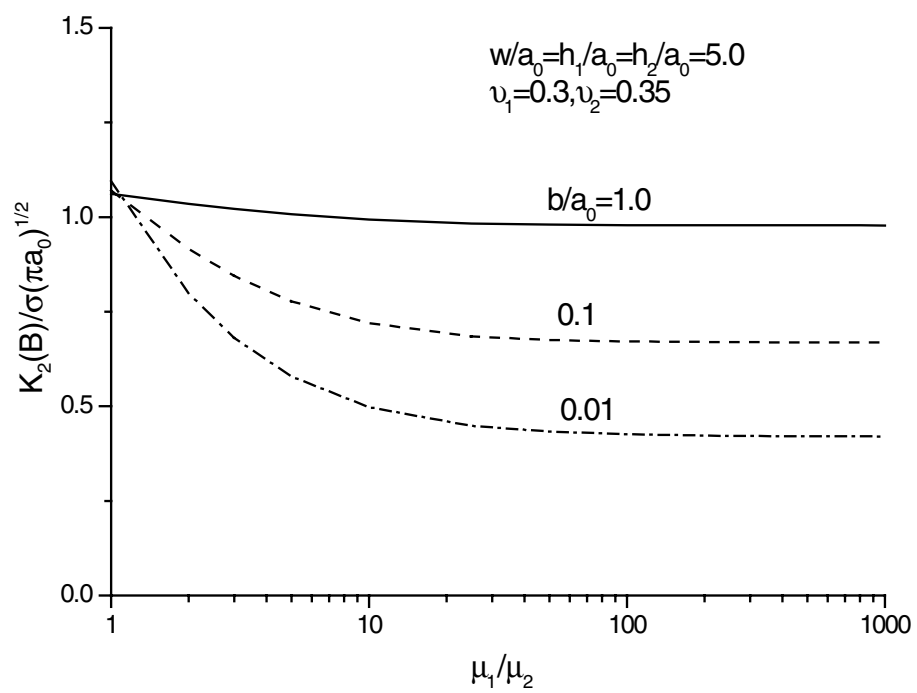

Fig. 25. Normalized stress intensity $K_{2}(B) / \sigma \sqrt{\pi a_{0}}$ versus $\mu_{1} / \mu_{2}$ for a finite bimaterial solid with different ratio of $b / a_{0}$, when the crack lies in a weaker material. 


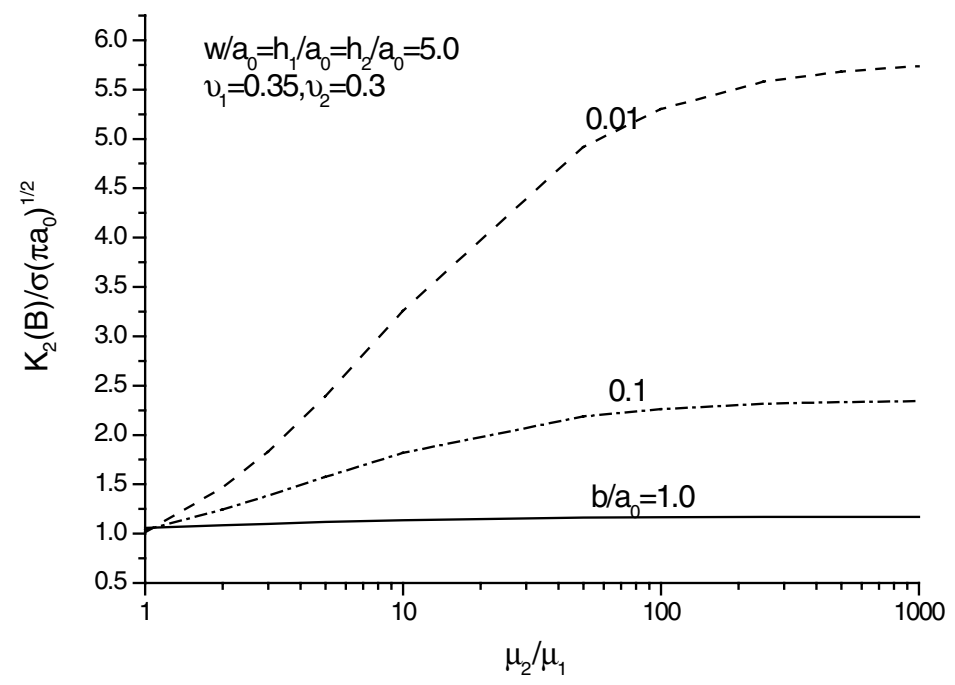

Fig. 26. Normalized stress intensity $K_{2}(B) / \sigma \sqrt{\pi a_{0}}$ versus $\mu_{2} / \mu_{1}$ for a finite bimaterial solid with different ratio of $b / a_{0}$, when the crack lies in a stiffer material.

\section{Conclusions}

- In the present paper, a crack perpendicular to the bimaterial interface of a finite solid is investigated analytically using the dislocation simulation approach and boundary collocation method.

- A crack perpendicular to the bimaterial interface of an infinite solid is solved also. For both the finite and infinite solids, the normal stress $\sigma_{x}$ ahead of the crack tip, which is near the interface, is characterized by the $K$ fields and the normal stress $\sigma_{y}$ is influenced by both the $K$ field and the $T$ stress in $0<r / b<0.5$ region for $b / a_{0}=0.01$; when $b / a_{0}=1.0$, the corresponding region is $0<r / b<0.1$ for both $\sigma_{x}$ and $\sigma_{y}$.

- As the crack approaches the interface, the parameter $b$ becomes an important length scale, which characterizes the dominated zone of the $K$ field and that of the $K$ field plus the $T$ stress field.

- The stress intensity factor becomes larger when the sample scale decreases. In a finite solid, the stress intensity factor is larger than that in an infinite solid.

- When the crack lies in a weak material, the stress intensity factor is smaller than that in the homogeneous material and the crack path is unstable when the crack tip is near the interface. When the crack lies in a stiffer material, the stress intensity factor is larger than that in the homogeneous material and the crack path is always stable with compressive $T$ stress, which is consistent with the experimental results given by Ståhle et al. (1995), Ye et al. (1992) and Cotterell and Rice (1980).

- The stress distributions for the finite and infinite solids of bimaterial are very similar but with the different stress intensity factors.

\section{Acknowledgements}

The authors wish to acknowledge helpful discussions with Prof. Ståhle, P. of Malmö University, Sweden. This work is supported by NSF of China (no. 10202023), Excellent Post-doctoral Research-Starting Fund of CAS. 


\section{References}

Bogy, D.B., 1971. On the plane elastic problem of a loaded crack terminating a material interface. Int. J. Fract. 38, 911-918.

Chen, D.H., 1994. A crack normal to and terminating at a bimaterial interface. Engng. Fract. Mech. 49, 517-523.

Chen, Y.Z., 2000. Closed form solutions of $T$-stress in plane elasticity crack problems. Int. J. Solids Struct. 37, 1629-1637.

Cook, T.S., Erdogan, F., 1972. Stress in bounded material with a crack perpendicular to the interface. Int. J. Engng. Sci. $10,677-697$.

Cotterell, B., Rice, J.R., 1980. Slightly curved or kinked cracks. Int. J. Fract. 16, 155-169.

Dundurs, J., 1969. In: Mura, T. (Ed.), Mathematics of Dislocation. ASME, New York, pp. 70-115.

Erdogan, F., Biricikoglu, B., 1973. Two bonded half plane with a crack through the interface. Int. J. Engng. Sci. 11, 745-766.

Gladwell, G.M.L., 1980. Contact Problem in the Classical Theory of Elasticity. Sijthoff \& Noordhoff., pp. 61-64.

Leblond, J.B., Frelat, J., 2000. Crack kinking from an initially closed crack. Int. J. Solids Struct. 37, 1595-1614.

Lin, K.Y., Mar, J.W., 1976. Finite element analysis of a stress intensity factors for crack at a bimaterial interface. Int. J. Fract. 12, 521531.

Meguid, S.A., Tan, M., Zhu, Z.H., 1995. Analysis of crack perpendicular to bimaterial interface using a novel finite element. Int. J. Fract. 75, 1-25.

Ståhle, P., Jens, G., Delfin, P., 1995. Crack path in a weak elastic layer covering a beam. Acta Mech. Solida Sinica 8, $579-583$.

Suo, Z.G., 1989. Singularities interacting with interface and cracks. Int. J. Solids Struct. 25, 1133-1142.

Wang, T.C., Ståhle, P., 1998a. Stress state in front of a crack perpendicular to bimaterial interface. Engng. Fract. Mech. 59, 471-485.

Wang, T.C., Ståhle, P., 1998b. A crack perpendicular to and terminating at a bimaterial interface. Acta Mech. Sinica 14 (1), 27-36.

Wang, W.C., Chen, J.T., 1993. Theoretical and experimental re-examination of a crack at a bimaterial interface. J. Strain Anal. 28, 53-61.

Ye, T., Suo, Z., Evans, A.G., 1992. Thin film cracking and the roles of substrate and interface. Int. J. Solids Struct. 29 (21), $2639-2648$.

Zak, A.R., Williams, M.L., 1963. Crack point singularities at a bimaterial interface. J. Appl. Mech. 30, 142-143. 